\title{
Divergent projections of the paraventricular nucleus of the thalamus mediate the selection of reactive and proactive defensive behaviors
}

Mario Penzo ( $\triangle$ mario.penzo@nih.gov )

National Institute of Mental Health https://orcid.org/0000-0002-5368-1802

Jun Ma

National Institute of Child Health and Human Development

Johann du Hoffmann

National Institute of Mental Health

Morgan Kindel

National Institute of Mental Health

\section{B. Sofia Beas}

National Institute of Mental Health

\section{Yogita Chudasama}

National Institute of Mental Health https://orcid.org/0000-0003-3349-8477

\section{Article}

Keywords: Paraventricular thalamus, active avoidance, fear conditioning, nucleus accumbens, central amygdala.

Posted Date: April 1st, 2021

DOl: https://doi.org/10.21203/rs.3.rs-322756/v1

License: (9) This work is licensed under a Creative Commons Attribution 4.0 International License. Read Full License

Version of Record: A version of this preprint was published at Nature Neuroscience on August 19th, 2021. See the published version at https://doi.org/10.1038/s41593-021-00912-7. 
1 Divergent projections of the paraventricular nucleus of the thalamus mediate the

2 selection of reactive and proactive defensive behaviors

3 Jun Ma ${ }^{1}$, Johann du Hoffmann ${ }^{2}$, Morgan Kindel ${ }^{1}$, B. Sofia Beas ${ }^{1}$, Yogita Chudasama ${ }^{2,3}$,

4 Mario A. Penzo ${ }^{1 \#}$

5

61 - Unit on the Neurobiology of Affective Memory, National Institute of Mental Health,

7 Bethesda, MD, USA.

82 - Rodent Behavioral Core, National Institute of Mental Health, National Institutes of

9 Health, Bethesda, MD, USA.

103 - Section on Behavioral Neuroscience, National Institute of Mental Health, National

11 Institutes of Health, Bethesda, MD, USA.

12

13

14 \# Correspondence

15 Mario A. Penzo, Ph.D. Unit on the Neurobiology of Affective Memory, National Institute

16 of Mental Health, Bethesda, MD 20850. Email:mario.penzo@nih.gov

17

18

\section{KEYWORDS}

20 Paraventricular thalamus, active avoidance, fear conditioning, nucleus accumbens, 21 central amygdala. 


\section{ABSTRACT}

The appropriate selection of reactive and proactive defensive behaviors amid fearful situations is essential for survival. Studies in both rodents and primates have shown that reactive defensive responses depend on the activity of the central nucleus of the amygdala $(\mathrm{CeA})$ whereas proactive ones primarily rely on the nucleus accumbens (NAc). However, the mechanisms underlying flexible switching between CeA-driven (reactive) and NAc-driven (proactive) defensive responses, remain unknown. Here, using a behavioral task in which mice must trade a reactive defensive strategy (i.e. freezing) for an instrumental one (i.e. active avoidance) to avoid punishment, we discovered that the paraventricular nucleus of the thalamus (PVT) mediates the selection of defensive behaviors through its interaction with the CeA and the NAc. In vivo calcium imaging using fiber photometry showed that unlike the PVT-CeA pathway which drives conditioned freezing responses, the PVT-NAc pathway is inhibited during freezing and instead signals active avoidance events. In addition, optogenetic manipulations of these circuits revealed that activity in the PVT-CeA or PVT-NAc pathway biases behavior toward the selection of reactive or proactive defensive responses, respectively. Our collective findings provide direct evidence that the PVT, a structure increasingly considered as a potential site for guiding behavioral decisions amid motivational conflicts, mediates flexible switching between opposing defensive behaviors. 


\section{INTRODUCTION}

The use of Pavlovian conditioning paradigms has allowed scientists to achieve substantial knowledge of the neuronal circuits and cellular processes underlying fear conditioning and its associated Pavlovian fear reactions (e.g., freezing, flight $)^{1-4}$. In contrast, the mechanisms controlling instrumental (proactive) defensive responses such as active avoidance and those that guide the selection of reactive and proactive defensive behaviors, are far less studied ${ }^{5-8}$. This is surprising considering that in nature animals readily engage and switch between both types of defensive strategies depending on threat imminence ${ }^{8-10}$.

While studies on the neurobiology of defensive behaviors have pointed to the contributions of both cortical and subcortical networks to the expression of specific defensive behaviors ${ }^{8,10}$, it is generally recognized that Pavlovian reactions such as freezing and conditioned flight are driven by genetically-defined neuronal subpopulations of the $\mathrm{CeA}^{4,11,12}$ and that proactive defensive behaviors largely depend on the $\mathrm{NAc}^{13-15}$. Interestingly, reports from the last decade suggest that these two regions of the brain likely compete for the control of defensive behaviors ${ }^{13,16}$. Specifically, these studies demonstrated that while lesions and pharmacological manipulations of the CeA attenuate freezing and promote active avoidance behavior $^{16,17}$, inactivation of the NAc decreases avoidance but enhances freezing ${ }^{13,14}$. Despite these seminal observations, the mechanisms dictating the balance of the competition between these two regions and behavioral outcomes remain unknown.

A potential candidate for mediating the selection of these two types of defensive behaviors is the basolateral amygdala (BLA) which sends projections to both the NAc 
67 and the $\mathrm{CeA}^{18}$. Indeed, while BLA projections to the $\mathrm{CeA}$ are known to mediate

82 conditioned freezing responses ${ }^{19}$, their projections to the NAc have been shown to support active avoidance behavior ${ }^{14}$. However, studies show that the necessity of the BLA for the expression of conditioned freezing and avoidance behaviors decreases with the passage of time ${ }^{20-22}$, suggesting that other brain regions likely contribute to arbitrating between these two defensive strategies. We predicted that the posterior portion of the PVT (pPVT) could be implicated in this process, considering its strong innervation of the $\mathrm{CeA}$ and the $\mathrm{NAc}^{23,24}$ as well as its documented role in mediating conditioned freezing responses, particularly at remote timepoints ${ }^{12,22}$. Consistent with this prediction, here we show that the PPVT drives active avoidance through its projections to the NAc. Moreover, we demonstrate that divergent projections from the pPVT to the NAc and CeA mediate the selection of active avoidance and freezing behavior, respectively. These findings highly the existence of a previously unrecognized switch for the selection of reactive and proactive defensive behaviors in the midline thalamus.

\section{RESULTS}

\section{PPVT ${ }^{\mathrm{D} 2 \mathrm{R}}$ neurons are inhibited during freezing}

The posterior PVT ( $p P V T$ ), is a stress-sensitive region of the thalamus that sends robust, largely non-overlapping projections to the $\mathrm{CeA}$ and the NAc (Extended Data Fig. $1)^{25}$. Unlike primates and carnivores, the rodent thalamus is largely devoid of GABAergic neurons ${ }^{26}$. Thus, projection neurons of the PVT are predominantly 
glutamatergic ${ }^{27}$. Although activation of CeA-projecting pPVT neurons is thought to be necessary for the retrieval of conditioned fear memory and its accompanying of freezing behavior $^{12,22}$, the NAc is the main target of most pPVT neurons (Extended Data Fig. $1 \mathrm{~h})^{25}$. Surprisingly, the contribution of pPVT-NAc neurons to fear-related behaviors is currently unknown. To investigate this, we first monitored the activity of dopamine D2 receptor expressing neurons of the pPVT ( $\left.p P V T^{\mathrm{D} 2 \mathrm{R}}\right)$ - a marker of stress-sensitive PVT neurons, the majority of which project to the NAc (Extended Data Fig. 1g-j) ${ }^{28-30}$ - during fear conditioning (Extended Data Fig. 2, 3, 4). Towards this goal, we expressed the genetically encoded calcium sensor GCaMP6s in pPVT ${ }^{\mathrm{D} 2 \mathrm{R}}$ neurons of $\mathrm{Drd2}$-Cre mice (Extended Data Fig. 2a). Next, following a habituation session (Day 0), on Day 1 mice were fear conditioned to an auditory cue (conditioned stimulus; CS) that co-terminated with a footshock (unconditioned stimulus; US), and on Day 2 a fear memory retrieval test was performed (Extended Data Fig. 2b). On both days, bulk changes in GCaMP6s fluorescence in $\mathrm{pPVT}{ }^{\mathrm{D} 2 \mathrm{R}}$ neurons were monitored using fiber photometry. We reasoned that since most pPVT ${ }^{\mathrm{D} 2 \mathrm{R}}$ neurons project to the NAc, bulk GCaMP6s fluorescence gathered from this neuronal population with fiber photometry would likely be dominated by the activity of PVT-NAc neurons. Consistent with previous reports, $P P V T^{\mathrm{D} 2 \mathrm{R}}$ neurons were readily activated by the US during the conditioning session on Day $1^{28,29}$ (Extended Data Fig. 2g, j, n). Notably, we also observed the emergence of small but consistent CS-evoked GCaMP6s responses in late conditioning trials, suggesting that the activity of these neurons is modulated by learning ${ }^{22}$ (Extended Data Fig. 2l, m). On Day 2, fear memory retrieval was associated with an average decrease in GCaMP6s fluorescence upon CS presentation (Extended Data Fig. 2h, k). These inhibitory 
112 GCaMP6s responses were variable and apparent in many but not all CS presentations

113 (Extended Data Fig. 3a), suggesting that they could be tied to behavior. To explore this

114 possibility, we classified all retrieval trials into three groups based on the percentage of

115 freezing displayed and quantified the average CS-evoked response for each trial

116 category (Extended Data Fig. 3a-d) (See Methods). As predicted, the average change

117 in GCaMP6s fluorescence for each trial category varied as a function of freezing

118 behavior, with the highest freezing scores giving rise to the most robust inhibitory

119 responses (Extended Data Fig. 3a-d). Notably, we did not observe any effect of retrieval 120 trial number on the GCaMP6s signal (Extended Data Fig. 4). These results indicate the

121 existence of an inverse relationship between freezing behavior and $p P V T^{\mathrm{D} 2 \mathrm{R}}$ neuron

122 activity. Consistent with this, pairwise comparisons of CS-evoked movement and

123 GCaMP6s fluorescence uncovered a modest but positive correlation between these two

124 parameters (Extended Data Fig. 3c). Importantly, no such correlation was observed on

125 imaging data gathered during habituation, prior to fear conditioning (Extended Data Fig.

$1263 e, f)$. As such, our collective results demonstrate that conditioned freezing is

127 associated with decreased activity among pPVT ${ }^{\mathrm{D} 2 \mathrm{R}}$ neurons.

$128 \quad$ PPVT $^{\mathrm{D} 2 \mathrm{R}}$ neurons signal active avoidance

The emergence of a positive correlation between the calcium signal of $p P V T^{D 2 R}$

130 neurons and movement following fear conditioning, likely reflects an overall link

131 between the activity of this neuronal population and the selection of active coping

132 strategies during fearful situations. To formally assess this possibility, we trained mice in

133 a two-way signaled active avoidance task (2AA) where performing a specific action -

134 moving to the neighboring compartment of a shuttle box - upon presentation of the CS, 
135 enables mice to avoid a footshock (US) (Fig. 1b). In well-trained mice, CS-related responses typically involve trading reactive (freezing) responses in favor of proactive

137 (instrumental) behavior (Fig. 1c) ${ }^{6}$. Still, animals engage in both freezing and avoidance 138 behavior on a trial-by-trial basis (Extended Data Fig. 5c, d). As such, this task provides 139 an opportunity for assessing neuronal activity while animals transition between reactive 140 and instrumental fear-related behavioral responses within single test sessions.

141 Consistent with previous reports, mice displayed prominent freezing behavior to the CS 142 in early sessions of the 2AA $\operatorname{task}^{6}$ (Fig. 1c) (Extended Data Fig. 5a). However, additional 143 training led to progressive decreases in CS-evoked freezing and a concomitant increase 144 in active avoidance responses (Fig. 1c) (Extended Data Fig. 5a). After three sessions 145 of $2 A A$ training (Days 1-3), $p P V T^{\mathrm{D} 2 R}$ neurons were imaged using fiber photometry 146 during two additional training sessions (Days 4 and 5) (Fig. 1c). Imaging trials were 147 subsequently divided into two behaviorally relevant categories: trials in which mice 148 avoided the US (avoidance) and trials in which they failed to do so (failure). Notably, 149 during failure trials, mice reliably shuttled to the adjacent compartment in response to 150 the US (Supplementary Movie 1). The data for avoidance and failure trials are 151 summarized in Figure 1 and Extended Data Figure 5 (Fig. 1d-h) (Extended Data Fig. $1525 b-d)$. Classifying trials in this manner revealed important differences in the activity of $153 \mathrm{pPVT}^{\mathrm{D} 2 \mathrm{R}}$ neurons during avoidance and failure trials (Supplementary Movie 1). First, 154 while CS presentations elicited robust increases in GCaMP6s fluorescence in PPVT ${ }^{\mathrm{D} 2 R}$ 155 neurons during avoidance trials, failures were mostly associated with strong decreases 156 in the activity of these neurons (Fig. 1g). In addition, presentation of the US during 157 failure trials was associated with robust activation of $\mathrm{PPVT}{ }^{\mathrm{D} 2 \mathrm{R}}$ neurons, consistent with 
158 our fear conditioning data as well as with previous reports (Fig. 1d, e) $)^{28,29}$. Interestingly, 159 whereas avoidance trials were by definition accompanied by instrumental responses, mice spent significantly more time freezing in response to the CS during failure trials 161 (Fig. 1f). This suggests that the differential dynamics of calcium signals of pPVTD2R 162 neurons observed across these trial categories could be related to behavior. To 163 investigate this possibility, we analyzed calcium transients time-locked to discrete task 164 and behavioral events such as CS onset, maximum velocity and freezing behavior (Fig. 165 1h). These analyses revealed that while CS onset, maximum velocity and escape 166 behavior (shuttling in response to the CS or US onset) were all associated with 167 increases in GCaMP6s fluorescence, freezing behavior was accompanied by 168 attenuated pPVT ${ }^{\mathrm{DR}}$ neuronal activity for both avoidance and failure trials (Fig. 1h).

169 Thus, while mean signal variations more accurately distinguish avoidance and failure 170 trials (Fig. 1f, g), discrete fluctuations in calcium transients largely answers to moment171 to-moment variation in behavior and not trial type (Fig. 1h). It is interesting that rises in 172 neuronal activity were correlated with maximum velocity particularly for avoidance trials 173 (Fig. 1h). This result indicates a potential relationship between pPVT ${ }^{\mathrm{D} 2 \mathrm{R}}$ neuron activity 174 and vigor, such as that necessary to avoid or escape an impending thread. Collectively, 175 our findings support the idea that, at the population level, $\mathrm{PPVT} \mathrm{T}^{\mathrm{D} 2 \mathrm{R}}$ neurons signal 176 instrumental defensive behaviors and are suppressed during freezing.

177 To investigate whether activation of $\mathrm{PPVT}{ }^{\mathrm{D} 2 \mathrm{R}}$ neurons is required for active 178 avoidance, we expressed in these neurons the inhibitory opsin eNpHR3.0 179 (Halorhodopsin; Halo) and trained mice in the 2AA task as described above (Fig. 1i) 180 (Extended Data Fig. 6). After 2AA training, mice were subjected to three test sessions, 
181 Test Days 1-3 (Fig. 1j-I). On Test Day 1 (light off), both control and Halo-expressing 182 mice displayed robust avoidance behavior (Fig. 1j, k). On Test Day 2 (light on), light 183 stimulation during CS presentations significantly attenuated the avoidance rate of Haloexpressing but not control mice (Fig. 1j, k). This effect was accompanied by a significant increase in the latency to avoid and an increase in CS-evoked freezing behavior (Fig. 1k, I). These parameters, partially recovered on Test Day 3 (light off) (Fig. 1j-I). Importantly, restricting optogenetic inhibition of $p P V T^{\mathrm{D} 2 \mathrm{R}}$ neurons to the inter-trial interval (ITI) did not significantly alter behavior (Extended Data Fig. 6k-t). Similarly, we 189 did not observe any light stimulation-induced effect on locomotion in an open field arena (Extended Data Fig. 6j). In summary, these findings show that, at the population level, 191 pPVT ${ }^{\mathrm{D} 2 \mathrm{R}}$ neurons are required for active avoidance behavior and are inhibited during 192 freezing.

\section{pPVT-NAc projections drive avoidance and antagonize freezing}

While most pPVT ${ }^{\mathrm{D} 2 \mathrm{R}}$ neurons project to the NAc (Extended Data Fig. 1$)^{29}$, it is unclear whether $\mathrm{PPVT}{ }^{\mathrm{D} 2 \mathrm{R}}-\mathrm{NAc}$ projections are modulated by passive and/or instrumental responding in the 2AA task. To address this question, we expressed the 197 genetically encoded calcium indicator GCaMP7s in pPVT ${ }^{\mathrm{D} 2 \mathrm{R}}$ neurons and implanted an optical fiber unilaterally in the NAc to measure the activity of pPVT ${ }^{\mathrm{D} 2 \mathrm{R}}-\mathrm{NAc}$ projections using fiber photometry (Fig. 2a-d) (Extended Data Fig. 7a). We trained GCaMP7sexpressing mice in the 2AA task, classified individual test trials as either avoidance or failure as described above, and analyzed the corresponding change in fluorescent signal associated with presentations of the CS (Fig. 2e-i) (Extended Data Fig. 7b). To 
204 were only trained for three days before imaging started (Fig. 2d) (Extended Data Fig.

205 7a). These analyses revealed that $P P V T^{D 2 R}-N A c$ projections were rapidly engaged

206 following CS presentations (Fig. 2i) and were robustly activated during avoidance and

207 escape episodes (Fig. 2i) irrespective of trial type. In contrast, CS-evoked freezing was

208 associated with a reduction in GCaMP7s fluorescence (Fig. 2i). These calcium

209 dynamics largely resembled those observed in pPVT ${ }^{\text {D2R }}$ neurons (Fig. 1d-h) (Extended

210 Data Fig. 5b). Together, these findings demonstrate that $P P V T^{D 2 R}-N A c$ projections

211 signal active avoidance and are suppressed during freezing behavior.

It is important to highlight that, unlike calcium responses recorded from the cell

213 bodies of $\mathrm{pPVT} \mathrm{T}^{\mathrm{D} R}$ neurons, for which increases were readily observed during shuttling

214 events (Fig. 1d), increases in GCaMP7 fluorescence in PPVT ${ }^{\mathrm{D} 2 \mathrm{R}}-\mathrm{NAc}$ terminals

215 appeared to emerge mostly at the conclusion of avoidance behavior (CS offset) (Fig.

$2162 \mathrm{e}, \mathrm{f})$. While alternative interpretations to this discrepancy are considered in the

217 Discussion section of this manuscript, one potential explanation is that calcium

218 transients accompanying avoidance behavior could result in weaker fluorescent

219 changes at pPVT ${ }^{\mathrm{D} 2 \mathrm{R}}-\mathrm{NAc}$ axon terminals, thereby limiting our ability to accurately

220 detect these with fiber photometry. To circumvent this limitation, we employed a viral

221 vector strategy that allowed us to record the cell bodies of NAc-projecting neurons

222 directly in the pPVT (Extended Data Fig. 8a, b). As with our axon terminal imaging

223 experiment, mice were first trained in the 2AA task for three sessions and then fiber

224 photometry imaging from the cell bodies of pPVT-NAc neurons was performed during

225 two additional sessions (Extended Data Fig. 8c, d). Notably, our results largely

226 resembled those obtained from pPVT ${ }^{\mathrm{D} 2 \mathrm{R}}$ neurons (Extended Data Fig. 8e-j). In 
227 particular, we noticed that while pPVT-NAc neurons were strongly recruited during avoidance trials, they were mostly suppressed during failures (Extended Data Fig. 8i). Moreover, pPVT-NAc neurons signaled active behavioral responses and were instead inhibited during freezing (Extended Data Fig. 8e, j) (Supplementary Movie 2). Together,

231 these findings demonstrate that pPVT projections to the NAc predominantly signal 232 active defensive responses.

Next, to investigate whether activation of pPVT-NAc projections is critical for 234 active avoidance, we optogenetically inhibited pPVT ${ }^{\mathrm{D} 2}-\mathrm{NAc}$ projections bilaterally 235 using Halo in a cohort of mice displaying robust avoidance behavior following training 236 (>70\% initial avoidance rate; See Methods) (Fig. 3a-c). Similar to the experiment 237 described above, following 2AA training (Fig. 3d-f) (Extended Data Fig. 9a) mice were 238 subjected to three test sessions (Test Days 1-3), and on Test Day 2 light stimulation 239 was delivered through optical fibers to silence $\mathrm{pPVT}{ }^{\mathrm{D} 2 \mathrm{R}}-\mathrm{NAc}$ communication during $\mathrm{CS}$ avoidance rate and increased the latency to avoid (Fig. 3g, h). In addition, a

242 concomitant increase in CS-evoked freezing was observed on Test Day 2 (Fig. 3i).

243 Importantly, pairwise comparison of light-evoked changes in freezing and avoidance 244 behavior across test sessions revealed an inverse relationship between these two 245 parameters (Extended Data Fig. 9c). Finally, restricting optogenetic inhibition of pPVT246 NAc projections to the ITI did not significantly alter behavior (Extended Data Fig. 10I-o).

247 Similarly, as with pPVT ${ }^{\mathrm{D} 2 \mathrm{R}}$ neurons, we did not observe any light stimulation-induced 248 effect on locomotion (Extended Data Fig. 10p). Together, these results demonstrate that 249 silencing $\mathrm{PPVT}^{\mathrm{D} 2 \mathrm{R}}-\mathrm{NAc}$ communication biases defensive behavioral responses towards 
reactive coping strategies. Notably, this effect is independent of the post-training avoidance rate, because similar observations were made in a cohort of mice in which the initial avoidance rate was markedly lower (Extended Data Fig. 10a-k). Moreover, our observation that optogenetic inhibition of pPVT-NAc projections on Test Day 2 lead to lasting behavioral effects on Test Day 3, indicates a potential role for this projection in the formation of persistent associations between fear cues and specific coping strategies.

\section{pPVT-CeA projections signal failure to avoid}

As described above, $\mathrm{pPVT}{ }^{\mathrm{D} 2 \mathrm{R}}$ neurons that project to the NAc are mostly distinct from those that project to the $\mathrm{CeA}$ (Extended Data Fig. 1) ${ }^{25}$. However, these anatomical differences do not necessarily implicate that functional distinctions exist between the two efferent pathways. Thus, to investigate how $\mathrm{pPVT}{ }^{\mathrm{D} 2 \mathrm{R}}-\mathrm{CeA}$ projections are modulated in the 2AA task, we expressed GCaMP7s in pPVT22R neurons of Drd2-Cre mice and implanted an optical fiber unilaterally in the CeA to measure the activity of pPVT ${ }^{\mathrm{D} 2}$-CeA projections with fiber photometry (Extended Data Fig. 11a-d). These experiments showed that unlike pPVT ${ }^{\mathrm{D} 2 \mathrm{R}}-\mathrm{NAc}$ terminals, $\mathrm{PPVT}{ }^{\mathrm{D} 2 \mathrm{R}}-\mathrm{Ce}$ e projections are not positively modulated by avoidance (Extended Data Fig. 11e-j). Surprisingly, CSevoked freezing behavior were not associated with significant changes in GCaMP7s fluorescence (Extended Data Fig. 11j). In contrast, pPVT ${ }^{\mathrm{D} 2 \mathrm{R}}$-CeA neurons were predominantly active during failure trials (Extended Data Fig. 11g, j), suggesting a link between engagement of $\mathrm{PPVT}{ }^{\mathrm{D} 2 \mathrm{R}}$-CeA projections and failure to avoid. Notably, previous studies have linked pPVT-CeA communication to the expression of conditioned fear - where Pavlovian reactions dominate behavior ${ }^{12,22}$. Altogether, these 
273 findings suggest the existence of functional differences between $P P V T^{\mathrm{D} 2 \mathrm{R}}-\mathrm{CeA}$ and

274 pPVT ${ }^{\mathrm{D} 2 \mathrm{R}}-\mathrm{NAc}$ projections, with the activity of pPVT ${ }^{\mathrm{D} 2 \mathrm{R}}-\mathrm{Ce}$ A projections increasing

275 during failures (when freezing dominates [Extended Data Fig. 11g]).

pPVT-CeA projections inhibit active avoidance

Previous reports demonstrated that active avoidance is under negative control by

278 the CeA, which controls Pavlovian reactions ${ }^{16,17}$. Specifically, these studies showed that

279 while blocking protein synthesis in the CeA promotes active avoidance ${ }^{17}$ lesions of this

280 region of the amygdala can unmask avoidance behavior in animals that initially fail to

281 avoid after $2 \mathrm{AA}$ training ${ }^{16}$. A major conclusion drawn from these studies is that CeA-

282 driven Pavlovian reactions compete with and thereby prevent the expression of

283 instrumental defensive behaviors. Because pPVT-CeA communication has been

284 previously shown to support conditioned freezing behavior ${ }^{12,22}$, we hypothesized that

285 attenuating pPVT-CeA projections could boost active avoidance. Consistent with this

286 prediction, optogenetically silencing pPVT-CeA projections using Halo diminished

287 freezing behavior and promoted avoidance behavior (Extended Data Fig. 12a-k).

288 Notably, this manipulation did not impact behavior when light simulation was restricted

289 to the ITI period (Extended Data Fig. 12I-o). Together, these results suggest that

290 silencing pPVT-CeA communication biases behavior away from reactive defensive

291 behavior and in favor of proactive ones.

Remarkably, Halo-mediated silencing of pPVT-CeA projections also promoted

293 active avoidance behavior in a subset of mice that failed to achieve $>30 \%$ avoidance

294 rate after the third 2AA session (See Methods) (Fig. 4) (Extended Data Fig. 13).

295 Consistent with previous manipulations, the increase in avoidance behavior was 
accompanied by a reduction in the latency to avoid and CS-evoked freezing (Fig. 4h, i). Moreover, pairwise comparisons revealed that consistent with the idea that in the 2AA task animals trade Pavlovian and instrumental defensive strategies, increases in active avoidance induced by silencing PPVT-CeA projections were linked to a reduction in freezing behavior (Extended Data Fig. 13c). Altogether, these results suggest that silencing pPVT-CeA communication biases behavior away from freezing and in favor of avoidance.

Our collective observations indicate that task-related activity within the pPVTNAc or the pPVT-CeA pathway biases defensive behavior towards active or passive responses, respectively. To investigate whether artificial stimulation of either pathway can generate behavioral bias, we expressed the red-shifted channelrhodopsin-2 variant ChimsonR in PPVTD2R neurons and implanted optical fibers bilaterally over the NAc or the CeA. Consistent with our model, we observed that while optogenetic stimulation of pPVT-NAc terminals potentiated, stimulation of pPVT-CeA projections diminished avoidance behavior (Extended Data Fig. 14). These results lend further support to our conclusion that divergent projections of the PPVT mediate the selection of opposing defensive behaviors.

\section{pPVT projections to the NAc and the CeA flexibly control the selection of}

\section{defensive behaviors}

A recurrent theme in our reported observations is the idea the pPVT projections to the NAc and the CeA can bias the selection of reactive and proactive defensive behaviors. But whether these projections flexibly control defensive behavior is unclear. To assess this, we selectively silenced pPVT projections to the NAc and the CeA in the 
same subjects across two individual test sessions (Fig. 5) (Extended Data Fig. 15). Notably, we found that while silencing pPVT-NAc projections impaired avoidance behavior (consistent with the above results), inhibition of pPVT-CeA terminals the following day fully restored active avoidance (Fig. 5e, f). Interestingly, an opposing but similarly dynamic modulation of freezing behavior was observed across sessions (Fig. 5g) (Extended Data Fig. 15d). In conclusion, these data demonstrate that the PPVT can flexibly bias defensive behaviors via the downstream circuitry described in the present study. Future studies should aim at identifying the local circuit dynamics and upstream mechanisms that guide the selection of defensive behaviors. As a proxy to this, using monosynaptic rabies tracing, we identified regions of the mouse brain that preferentially innervate pPVT-CeA or pPVT-NAc neurons (Extended Data Fig. 16).

\section{DISCUSSION}

In the present study, we have identified the PPVT as a key brain structure that mediates the selection of competing defensive strategies, namely Pavlovian reactions and instrumental behavior. These findings add to a growing body of literature that places the PVT as an important regulator of emotional and motivational processes ${ }^{31,32}$. Importantly, we uncovered that the anatomical and functional segregation of PVT projections to the $\mathrm{CeA}$ and the NAc underscores its role in biasing behavioral selection. Collectively, these findings expand on previous studies linking PVT function to reactive defensive responses ${ }^{12,22,28,33}$, provide a circuit mechanism by which opposing defensive strategies are leveraged ${ }^{8}$, and support the notion that the PVT is critical for the orchestration of adaptive behavioral responses $32,34,35$. 
Recent literature shows that the PPVT is predominantly sensitive to aversive

343 stimuli ${ }^{28,29}$. In turn, the pPVT appears to orchestrate adaptive responses that allow

344 animals to cope with ongoing demands (Penzo and Gao, in press). Consistent with this

345 view and in addition to its role in driving Pavlovian defensive reactions, the pPVT is now

346 recognized to promote goal-directed instrumental responses including food seeking,

347 drug seeking and maternal behaviors ${ }^{36-40}$. Importantly, some of these studies have

348 directly implicated NAc projections of the pPVT as guiding instrumental behaviors

349 during aversive states ${ }^{37-42}$. In light of these recent reports, our finding that the pPVT-

350 NAc pathway also regulates instrumental defensive behaviors suggests thar these

351 projections may generally promote goal-directed behaviors. From this perspective, the

352 pPVT-CeA pathway could support behavioral strategies that favor Pavlovian

353 responding irrespective of whether responses are reward oriented or defensive in

354 nature (Penzo and Gao, in press). Accordingly, it was recently proposed that pPVT-

355 CeA projections drive morphine-induced conditioned place preference likely through

356 Pavlovian incentive motivation ${ }^{43}$.

The pPVT is not the only brain region that innervates both the NAc and the CeA.

358 Indeed, the BLA and the insular cortex also send divergent projection to these

359 structures and these projections have been implicated in emotional and motivational

360 processes ${ }^{14,19,44-46}$. Particularly relevant to the present study is the notion that the BLA

361 has been shown to contribute to both conditioned freezing and active avoidance

362 behavior via its projections to the CeA and the NAc, respectively. This leads to the

363 question of what the differential contributions of the BLA and the PVT are to these

364 opposing defensive behaviors. One possibility discussed earlier in this manuscript is 
365 that the BLA's contribution to conditioned reactive and proactive defensive behaviors 366 decreases over time ${ }^{20-22}$. As such the PVT may increasingly become the dominant 367 pathway by which freezing and active avoidance behaviors are regulated. Alternatively, 368 and possibly in addition to this time dependent role, the PVT may serve to contextualize 369 aversive memories by integrating these with information about internal state (owing to 370 its strong innervation by the hypothalamus and the brainstem). Consistent with this idea, 371 the PVT has been recently proposed to arbiter amid motivational conflicts ${ }^{32}$. Future 372 studies should aim to identify the precise mechanisms by which the PVT regulates 373 behavior and how these differ from those of other regions known to regulate the same 374 behaviors. Emergent studies support the notion that the neuronal circuits of the PVT are 376 functionally diverse ${ }^{31}$. Indeed, we recently identified two major classes of PVT neurons 377 that can be distinguished on the basis of genetic, anatomic (connectional) and 378 functional differences ${ }^{29}$. Specifically, it was suggested that PVT neuronal subtypes 379 could be identified due to their expression, or lack thereof, of the Drd2 gene (and D2R 380 protein). Functionally, compared to D2R negative PVT neurons which at the population 381 level appear to be modulated by stimulus salience, D2R-expressing neurons are 382 primarily sensitive to aversive stimuli ${ }^{28,29}$. Our results expand on this recent report by 383 showing further anatomical and functional heterogeneity among aversive-sensitive 384 pPVT ${ }^{\mathrm{D} 2 \mathrm{R}}$ neurons. These findings should encourage future studies to classify PVT 385 neurons based on the intersection of genetic markers and projection target ${ }^{31}$. Similar 386 intersectional approaches have let to the discovery of functional segregation in other 387 brain areas $^{47-51}$. 
The observation that optogenetic inhibition of pPVT-NAc projections leads to lasting impairments in active avoidance behavior, is reminiscent of a previous report demonstrating that silencing pPVT-CeA projections produces similar lasting effects in 391 freezing behavior during fear memory retrieval ${ }^{22}$. In that study, the authors concluded 392 that the persistent reduction in freezing behavior indicated an overall role for this 393 pathway in controlling the maintenance of fear memories. Our findings are at odd with 394 this interpretation. Specifically, we found that while silencing pPVT-NAc projections 395 produces a lasting impairment in active avoidance, the same manipulation led to a 396 concomitant increment in CS-evoked freezing. As such, our findings do not support a conclusion in which persistent reductions in avoidance behavior resulting from pPVT398 NAc silencing are due to deficits in fear memory maintenance. Instead, in light of this previous report and our collective findings, we propose that the activation of pPVT 400 projections to the NAc and the CeA links fear (CS/US) associations to the selection of 401 specific defensive strategies. As such, our findings help remodel current views on the 402 role that the PVT plays in the formation and maintenance of aversive memories. 403 Subsequent studies in this topic should aim at identifying the local and upstream circuit 404 mechanisms that promote behavioral bias. which animals switch between two opposing defensive strategies, Pavlovian freezing, 407 and active avoidance. In nature, animals typically engage in these different behavioral strategies as a function of threat imminence ${ }^{8-10}$. From this perspective, active avoidance

409 is thought to have evolved as a way to deal with distant threats, whereas reactive behaviors mostly emerge during imminent threats ${ }^{10}$. Within this framework the idea that 
411 the 2AA task yields a subset of animals that fail to engage in avoidance behavior (low

412 avoiders), seems to suggest that this task might be ineffective for studying ethologically

413 relevant defensive behaviors. One potential explanation to the variability in behavioral

414 outcome, could be that some animals view the threat (US) as more imminent than

415 others, and thus display freezing behavior when avoidance is possible. Consistent with

416 this notion, a recent study demonstrated that reducing threat imminence by increasing

417 the duration of the CS virtually eliminates low avoiders ${ }^{52}$. Thus, whereas here we have

418 effectively exploited individual differences in animal performance to extract meaningful

419 information of the mechanisms that underlie behavioral selection, identifying

420 ethologically relevant behavioral models that allow for animals to engage in both

421 proactive and reactive defensive strategies as a function of threat imminence would be

422 of tremendous value to studies on the neurobiology of defensive behaviors.

424 REFERENCES

4251 LeDoux, J. E. Emotion circuits in the brain. Annu Rev Neurosci 23, 155-184,

426 doi:10.1146/annurev.neuro.23.1.155 (2000).

4272 Duvarci, S. \& Pare, D. Amygdala microcircuits controlling learned fear. Neuron 82, 966-980,

428 doi:10.1016/j.neuron.2014.04.042 (2014).

4293 Fanselow, M. S. \& Poulos, A. M. The neuroscience of mammalian associative learning. Annu Rev

$430 \quad$ Psychol 56, 207-234, doi:10.1146/annurev.psych.56.091103.070213 (2005).

4314 Fadok, J. P. et al. A competitive inhibitory circuit for selection of active and passive fear

432 responses. Nature 542, 96-100, doi:10.1038/nature21047 (2017).

4335 LeDoux, J. E., Moscarello, J., Sears, R. \& Campese, V. The birth, death and resurrection of

434 avoidance: a reconceptualization of a troubled paradigm. Mol Psychiatry 22, 24-36,

435 doi:10.1038/mp.2016.166 (2017).

4366 Cain, C. K. Avoidance Problems Reconsidered. Curr Opin Behav Sci 26, 9-17,

437 doi:10.1016/j.cobeha.2018.09.002 (2019).

4387 Krypotos, A. M., Effting, M., Kindt, M. \& Beckers, T. Avoidance learning: a review of theoretical

439 models and recent developments. Front Behav Neurosci 9, 189, doi:10.3389/fnbeh.2015.00189

440 (2015). 
4418 Mobbs, D., Headley, D. B., Ding, W. \& Dayan, P. Space, Time, and Fear: Survival Computations along Defensive Circuits. Trends Cogn Sci 24, 228-241, doi:10.1016/j.tics.2019.12.016 (2020).

9 Fanselow, M. S. \& Lester, L. S. in Evolution and learning. 185-212 (Lawrence Erlbaum Associates, Inc, 1988).

10 Mobbs, D. The ethological deconstruction of fear(s). Curr Opin Behav Sci 24, 32-37, doi:10.1016/j.cobeha.2018.02.008 (2018).

$11 \mathrm{Li}, \mathrm{H}$. et al. Experience-dependent modification of a central amygdala fear circuit. Nat Neurosci 16, 332-339, doi:10.1038/nn.3322 (2013).

12 Penzo, M. A. et al. The paraventricular thalamus controls a central amygdala fear circuit. Nature 519, 455-459, doi:10.1038/nature13978 (2015).

13 Bravo-Rivera, C., Roman-Ortiz, C., Brignoni-Perez, E., Sotres-Bayon, F. \& Quirk, G. J. Neural structures mediating expression and extinction of platform-mediated avoidance. J Neurosci 34, 9736-9742, doi:10.1523/JNEUROSCI.0191-14.2014 (2014).

14 Ramirez, F., Moscarello, J. M., LeDoux, J. E. \& Sears, R. M. Active avoidance requires a serial basal amygdala to nucleus accumbens shell circuit. J Neurosci 35, 3470-3477, doi:10.1523/JNEUROSCI.1331-14.2015 (2015).

15 Oleson, E. B., Gentry, R. N., Chioma, V. C. \& Cheer, J. F. Subsecond dopamine release in the nucleus accumbens predicts conditioned punishment and its successful avoidance. $J$ Neurosci 32, 14804-14808, doi:10.1523/JNEUROSCI.3087-12.2012 (2012).

16 Choi, J. S., Cain, C. K. \& LeDoux, J. E. The role of amygdala nuclei in the expression of auditory signaled two-way active avoidance in rats. Learn Mem 17, 139-147, doi:10.1101/Im.1676610 (2010).

17 Moscarello, J. M. \& LeDoux, J. E. Active avoidance learning requires prefrontal suppression of amygdala-mediated defensive reactions. J Neurosci 33, 3815-3823, doi:10.1523/JNEUROSCI.2596-12.2013 (2013).

18 Beyeler, A. et al. Organization of Valence-Encoding and Projection-Defined Neurons in the Basolateral Amygdala. Cell Rep 22, 905-918, doi:10.1016/j.celrep.2017.12.097 (2018).

19 Jimenez, S. A. \& Maren, S. Nuclear disconnection within the amygdala reveals a direct pathway to fear. Learn Mem 16, 766-768, doi:10.1101/Im.1607109 (2009).

20 Maren, S. Neurotoxic basolateral amygdala lesions impair learning and memory but not the performance of conditional fear in rats. J Neurosci 19, 8696-8703 (1999).

21 Poremba, A. \& Gabriel, M. Amygdala neurons mediate acquisition but not maintenance of instrumental avoidance behavior in rabbits. J Neurosci 19, 9635-9641 (1999).

22 Do-Monte, F. H., Quinones-Laracuente, K. \& Quirk, G. J. A temporal shift in the circuits mediating retrieval of fear memory. Nature 519, 460-463, doi:10.1038/nature14030 (2015).

23 Vertes, R. P. \& Hoover, W. B. Projections of the paraventricular and paratenial nuclei of the dorsal midline thalamus in the rat. J Comp Neurol 508, 212-237, doi:10.1002/cne.21679 (2008).

$24 \mathrm{Li}, \mathrm{S}$. \& Kirouac, G. J. Projections from the paraventricular nucleus of the thalamus to the forebrain, with special emphasis on the extended amygdala. J Comp Neurol 506, 263-287, doi:10.1002/cne.21502 (2008).

25 Dong, X., Li, S. \& Kirouac, G. J. Collateralization of projections from the paraventricular nucleus of the thalamus to the nucleus accumbens, bed nucleus of the stria terminalis, and central nucleus of the amygdala. Brain Struct Funct 222, 3927-3943, doi:10.1007/s00429-017-1445-8 (2017).

26 Arcelli, P., Frassoni, C., Regondi, M. C., De Biasi, S. \& Spreafico, R. GABAergic neurons in mammalian thalamus: a marker of thalamic complexity? Brain Res Bull 42, 27-37 (1997). 
Fremeau, R. T., Jr., VogImaier, S., Seal, R. P. \& Edwards, R. H. VGLUTs define subsets of excitatory neurons and suggest novel roles for glutamate. Trends Neurosci 27, 98-103, doi:10.1016/j.tins.2003.11.005 (2004).

28 Beas, B. S. et al. The locus coeruleus drives disinhibition in the midline thalamus via a dopaminergic mechanism. Nat Neurosci, doi:10.1038/s41593-018-0167-4 (2018).

29 Gao, C. et al. Two genetically, anatomically and functionally distinct cell types segregate across anteroposterior axis of paraventricular thalamus. Nat Neurosci 23, 217-228, doi:10.1038/s41593-019-0572-3 (2020).

30 Clark, A. M. et al. Dopamine D2 Receptors in the Paraventricular Thalamus Attenuate Cocaine Locomotor Sensitization. eNeuro 4, doi:10.1523/ENEURO.0227-17.2017 (2017).

31 McGinty, J. F. \& Otis, J. M. Heterogeneity in the Paraventricular Thalamus: The Traffic Light of Motivated Behaviors. Frontiers in Behavioral Neuroscience 14, doi:10.3389/fnbeh.2020.590528 (2020).

32 McNally, G. P. Motivational competition and the paraventricular thalamus. Neurosci Biobehav Rev, doi:10.1016/j.neubiorev.2021.02.021 (2021).

33 Li, Y., Dong, X., Li, S. \& Kirouac, G. J. Lesions of the posterior paraventricular nucleus of the thalamus attenuate fear expression. Front Behav Neurosci 8, 94, doi:10.3389/fnbeh.2014.00094 (2014).

34 Kirouac, G. J. Placing the paraventricular nucleus of the thalamus within the brain circuits that control behavior. Neurosci Biobehav Rev 56, 315-329, doi:10.1016/j.neubiorev.2015.08.005 (2015).

35 Choi, E. A. \& McNally, G. P. Paraventricular Thalamus Balances Danger and Reward. The Journal of neuroscience : the official journal of the Society for Neuroscience 37, 3018-3029 (2017).

36 Watarai, A. et al. The blockade of oxytocin receptors in the paraventricular thalamus reduces maternal crouching behavior over pups in lactating mice. Neurosci Lett 720, 134761, doi:10.1016/j.neulet.2020.134761 (2020).

37 Zhu, Y., Wienecke, C. F., Nachtrab, G. \& Chen, X. A thalamic input to the nucleus accumbens mediates opiate dependence. Nature 530, 219-222, doi:10.1038/nature16954 (2016).

38 Meffre, J. et al. Orexin in the Posterior Paraventricular Thalamus Mediates Hunger-Related Signals in the Nucleus Accumbens Core. Curr Biol, doi:10.1016/j.cub.2019.07.069 (2019).

39 Otis, J. M. et al. Paraventricular thalamus projection neurons integrate cortical and hypothalamic signals for cue-reward processing. Neuron 102, doi:https://doi.org/10.1016/j.neuron.2019.05.018. (2019).

40 Labouebe, G., Boutrel, B., Tarussio, D. \& Thorens, B. Glucose-responsive neurons of the paraventricular thalamus control sucrose-seeking behavior. Nat Neurosci 19, 999-1002, doi:10.1038/nn.4331 (2016).

41 Beas, B. S. et al. A ventrolateral medulla-midline thalamic circuit for hypoglycemic feeding. Nat Commun 11, 6218, doi:10.1038/s41467-020-19980-7 (2020).

42 Ong, Z. Y., Liu, J. J., Pang, Z. P. \& Grill, H. J. Paraventricular Thalamic Control of Food Intake and Reward: Role of Glucagon-Like Peptide-1 Receptor Signaling. Neuropsychopharmacology 42, 2387-2397, doi:10.1038/npp.2017.150 (2017).

43 Keyes, P. C. et al. Orchestrating Opiate-Associated Memories in Thalamic Circuits. Neuron 107, 1113-1123 e1114, doi:10.1016/j.neuron.2020.06.028 (2020).

$44 \quad$ Namburi, P. et al. A circuit mechanism for differentiating positive and negative associations. Nature 520, 675-678, doi:10.1038/nature14366 (2015).

45 Rogers-Carter, M. M., Djerdjaj, A., Gribbons, K. B., Varela, J. A. \& Christianson, J. P. Insular Cortex Projections to Nucleus Accumbens Core Mediate Social Approach to Stressed Juvenile Rats. J Neurosci 39, 8717-8729, doi:10.1523/JNEUROSCI.0316-19.2019 (2019). 
46 Schiff, H. C. et al. An Insula-Central Amygdala Circuit for Guiding Tastant-Reinforced Choice Behavior. J Neurosci 38, 1418-1429, doi:10.1523/JNEUROSCI.1773-17.2017 (2018).

47 Beier, K. T. et al. Circuit Architecture of VTA Dopamine Neurons Revealed by Systematic InputOutput Mapping. Cell 162, 622-634, doi:10.1016/j.cell.2015.07.015 (2015).

48 Heymann, G. et al. Synergy of Distinct Dopamine Projection Populations in Behavioral Reinforcement. Neuron 105, 909-920 e905, doi:10.1016/j.neuron.2019.11.024 (2020).

49 Kim, E. J., Juavinett, A. L., Kyubwa, E. M., Jacobs, M. W. \& Callaway, E. M. Three Types of Cortical Layer 5 Neurons That Differ in Brain-wide Connectivity and Function. Neuron 88, 1253-1267, doi:10.1016/j.neuron.2015.11.002 (2015).

50 Ren, J. et al. Anatomically Defined and Functionally Distinct Dorsal Raphe Serotonin Subsystems. Cell 175, 472-487 e420, doi:10.1016/j.cell.2018.07.043 (2018).

51 Wallace, M. L. et al. Genetically Distinct Parallel Pathways in the Entopeduncular Nucleus for Limbic and Sensorimotor Output of the Basal Ganglia. Neuron 94, 138-152 e135, doi:10.1016/j.neuron.2017.03.017 (2017).

52 Laughlin, L. C., Moloney, D. M., Samels, S. B., Sears, R. M. \& Cain, C. K. Reducing shock imminence eliminates poor avoidance in rats. Learn Mem 27, 270-274, doi:10.1101/Im.051557.120 (2020).

53 Haynes, K., Fearnhead, P. \& Eckley, I. A. A computationally efficient nonparametric approach for changepoint detection. Stat Comput 27, 1293-1305, doi:10.1007/s11222-016-9687-5 (2017).

\section{FIGURE LEGENDS}

Figure 1. $\mathrm{PPVT}^{\mathrm{D} 2 \mathrm{R}}$ neurons control active avoidance and are inhibited during

\section{freezing.}

a, Left: Representative image from a mouse expressing GCaMP6s in $\mathrm{PPVT}^{\mathrm{D} 2 \mathrm{R}}$ neurons of Drd2-Cre mice and implanted with an optical fiber in the pPVT. Right: Optical fiber placements ( $n=5$ mice). b, Schematic of the 2AA task. c, Avoidance Rate (black) and freezing time during the CS (red) across training (Days 1-3) and test sessions (Days 45). Fiber photometry imaging sessions are depicted by the blue lines. d, Representative imaging traces from a sample subject. Individual imaging traces for avoidance (blue) and failure (red) trials. Black square pulses depict the duration of each CS presentation. Yellow and red backgrounds show the maximal duration of CS and US presentation, respectively. e, Top: Heatmaps showing individual calcium responses for all avoidance 
567 and failure trials recorded. Bottom: Average calcium signal and CS duration of the upper panels (Avoidance trials, blue; failure trials, red; CS duration, black). f, Freezing time during the CS (top) in avoidance trials is significantly less than failure trials, while the peak (middle) and mean (bottom) of calcium signals during the CS is significantly larger in avoidance trials. Avoidance, $n=118$ Trials; Failure, $n=122$ Trials; two-tailed Student's $t$-test. ${ }^{* * *} P<0.001$. g, Quantification of calcium signal during pre-CS, CS and post-CS for avoidance (blue) and failure trials (red). AUC, One-way ANOVA followed by two-stage linear step-up procedure of Benjamini, Krieger and Yekutieli. Avoidance: $n=$ 118 Trials; $F(2,351)=75.89$; group comparisons, pre-CS vs $C S^{* * *} P<0.001$, pre-CS vs post-CS ${ }^{*} P=0.038$, CS vs post-CS ${ }^{* * *} P<0.001$. Failure: $\mathrm{n}=122$ Trials; $\mathrm{F}(2$,

$577363)=66.54 ;$ group comparisons, ${ }^{* * *} P<0.001$. h, Left: Averaged calcium responses

578 during CS onset, CS maximal velocity, escape initiation, escape and CS freezing 579 epochs for all avoidance (blue) and failure (red) trials. Right: quantification of calcium 580 signal in each epoch duration. AUC, two-tailed Student's $t$-test. CS: Avoidance, $n=118$

581 Events; Failure, $\mathrm{n}=122$ Events; $P=0.74$. Max. Velocity: Avoidance, $\mathrm{n}=117$ Events;

582 Failure, $\mathrm{n}=122$ Events; ${ }^{*} P=0.024$. Escape initiate: Avoidance, $\mathrm{n}=113$ Events; Failure, 583 $\mathrm{n}=115$ Events; ${ }^{* \star *} P<0.001$. Escape: Avoidance, $\mathrm{n}=118$ Events; Failure, $\mathrm{n}=122$

584 Events; ${ }^{* \star} P=0.0064$. Freezing: Avoidance, $\mathrm{n}=92$ Events; Failure, $\mathrm{n}=157$ Events; $P=$ 585 0.98. $\mathbf{i}$, Schematic of the viral vector strategy for optogenetic silencing of $P P V T^{\mathrm{D} 2 \mathrm{R}}$ 586 neurons. j-l, Left: Optogenetic inhibition of pPVT ${ }^{\mathrm{D} 2 R}$ neurons significantly decreases 587 avoidance rate (j), while increases latency to avoid $(k)$ and freezing time during the CS 588 (I). Right: Normalization of the Day 1 in each group. Ctl, $\mathrm{n}=8$ mice; Halo, $\mathrm{n}=9$ mice, 589 two-way ANOVA followed by two-stage linear step-up procedure of Benjamini, Krieger 
and Yekutieli. Avoidance Rate: $F(2,30)=5.48$; group comparisons, Halo, Day 1 vs Day $2{ }^{* * *} P<0.001$, Day 1 vs Day $3 P=0.06$. Latency to avoid: $F(2,30)=2.48$; group comparisons, Halo, Day 1 vs Day $2{ }^{* *} P=0.0037$. CS freezing: $F(2,30)=0.19$; group comparisons, Halo, Day 1 vs Day $2{ }^{*} P=0.04$. Optogenetic inhibition sessions are depicted by the yellow background. Data shown as mean \pm s.e.m.

\section{Figure 2. $\mathrm{PPVT}^{\mathrm{D} 2 \mathrm{R}}-\mathrm{NAc}$ axon terminals signal active avoidance.}

a, Schematic of the viral vector strategy and optical fiber placement used for fiber photometry imaging of $\mathrm{PPVT}^{\mathrm{D} 2 \mathrm{R}}-\mathrm{NAc}$ axon terminals. $\mathbf{b}$, Representative images from a mouse expressing GCaMP7s in pPVT ${ }^{\mathrm{D} 2 \mathrm{R}}$ neurons and implanted with an optical fiber in the NAc. c, Optical fiber placements $(n=4$ mice). d, Avoidance Rate (black) and freezing time during the CS (red) across all training and test sessions. Fiber photometry imaging sessions are depicted by the blue lines. e, Representative imaging traces from a sample subject. Individual imaging traces for avoidance (blue) and failure (red) trials.

Black square pulses depict the duration of each CS presentation. Yellow and red backgrounds show the maximal duration of CS and US presentation, respectively. $\mathbf{f}$, Top: Heatmaps showing individual calcium responses for all avoidance and failure trials. Bottom: Average calcium signal and CS duration of the upper panels (Avoidance trials, blue; failure trials, red; CS duration, black). g, Freezing time during the CS (top) in avoidance trials is significantly lower compared to failure trials, while the peak (middle) and mean (bottom) values of the calcium signal during the CS are similar for both groups. Two-tailed Student's $t$-test. Avoidance, $n=80$ Trials. Failure, $n=130$ Trials. Freezing, ${ }^{* *} P<0.001$. Peak Signal, $P=0.14$. Mean Signal, $P=0.75$. h, Quantification 
613 of calcium signal during pre-CS, CS and post-CS periods for avoidance (blue) and

614 failure (red) trials. AUC, One-way ANOVA followed by two-stage linear step-up

615 procedure of Benjamini, Krieger and Yekutieli. Avoidance: $n=80$ Trials, $F(2$,

$616237)=4.37$; group comparisons, pre-CS vs $C S{ }^{* *} P=0.0065$, pre-CS vs post-CS $P=$

$6170.67, \mathrm{CS}$ vs post-CS ${ }^{*} P=0.021$. Failure: $\mathrm{n}=130$ Trials, $\mathrm{F}(2,387)=121.2$; group

618 comparisons, pre-CS vs CS ${ }^{* *} P<0.001$, pre-CS vs post-CS $P=0.072$, CS vs post-CS

$619{ }^{* * *} P<0.001$. i, Left: Averaged calcium responses during CS onset, CS maximal

620 velocity, escape initiation, escape and CS freezing epochs for all avoidance (blue) and

621 failure (red) trials. Right: Quantification of calcium signal in each epoch duration. AUC,

622 two-tailed Student's $t$-test. CS: Avoidance, $n=80$ Events; Failure, $n=130$ Events; $P=$

623 0.17. Max. Velocity: Avoidance, $n=80$ Events; Failure, $n=129$ Events; ${ }^{* * *} P<0.001$.

624 Escape initiate: Avoidance, $\mathrm{n}=79$ Events; Failure, $\mathrm{n}=123$ Events; $P=0.74$. Escape:

625 Avoidance, $\mathrm{n}=80$ Events; Failure, $\mathrm{n}=130$ Events; $P=0.78$. Freezing: Avoidance, $\mathrm{n}=$

62676 Events; Failure, $\mathrm{n}=175$ Events; $P=0.78$. Data shown as mean \pm s.e.m.

627

628

629

Figure 3. Optogenetic inhibition of $\mathrm{pPVT}^{\mathrm{D} 2 \mathrm{R}}-\mathrm{NAc}$ axon terminals decreases active avoidance and increases freezing.

630 a, Schematic of the viral vector strategy and optical fiber placement for optogenetic

631 silencing of $p P V T^{\mathrm{D} 2 \mathrm{R}}-\mathrm{NAc}$ axon terminals in the 2AA task. b, Representative images

632 from a mouse expressing Halo-mCherry in pPVT ${ }^{\mathrm{D} 2 \mathrm{R}}$ neurons (left) and implanted with

633 optical fibers in the NAc (right). c, Optical fiber placements ( $n=8$ mice per group). d-f,

634 Avoidance rate (d), latency to avoid (e) and freezing time during the CS (f) across all

635 training sessions for both Ctl (black) and Halo (red) groups. g-i, Left: Optogenetic 
636 inhibition of pPVT ${ }^{\mathrm{D} 2 \mathrm{R}}-\mathrm{NAc}$ axon terminals persistently decreases avoidance rate $(\mathrm{g})$,

637 while increases latency to avoid (h) and freezing time during the CS (i). Right:

638 Normalization of the Day 1 in each group. Two-way ANOVA followed by two-stage

639 linear step-up procedure of Benjamini, Krieger and Yekutieli. Avoidance rate: $F(2$,

$64028)=18.04$; group comparisons, Halo, Day 1 vs Day $2{ }^{* * *} P<0.001$, Day 1 vs Day 3

$641{ }^{* * *} P<0.001$. Latency to avoid: $F(2,28)=5.63$; group comparisons, Ctl, Day 1 vs Day 3

$642{ }^{*} P=0.028$; Halo, Day 1 vs Day $2{ }^{* * *} P<0.001$, Day 1 vs Day $3{ }^{* \star *} P<0.001$. CS

643 Freezing: $F(2,28)=1.45$; group comparisons, Halo, Day 1 vs Day $2{ }^{*} P=0.049$, Day 1

644 vs Day $3{ }^{* *} P=0.0092$. Optogenetic inhibition session is depicted by the yellow

645 background. Data shown as mean \pm s.e.m.

646

647 Figure 4. Optogenetic inhibition pPVT ${ }^{\mathrm{D} 2}$-CeA axon terminals increases active 648 avoidance and reduces freezing.

649 a, Schematic of the viral vector strategy and optical fiber placement used for

650 optogenetic silencing of pPVT ${ }^{\mathrm{D} 2 \mathrm{R}}-\mathrm{Ce} A$ axon terminals in the $2 \mathrm{AA}$ task. $\mathbf{b}$,

651 Representative images from a mouse expressing Halo-mCherry in pPVT ${ }^{\mathrm{D} 2 \mathrm{R}}$ neurons

652 and implanted with optical fibers in the CeA. c, Optical fiber placements $(n=9$ mice per

653 group). d-f, Avoidance rate (d), latency to avoid (e) and freezing time during the CS (f)

654 across all training days in both Ctl (black) and Halo (red) groups. g-i, Top: Optogenetic

655 inhibition of $p P V T^{\mathrm{D} 2 \mathrm{R}}-\mathrm{CeA}$ axon terminals gradually increases avoidance rate $(\mathrm{g})$ and

656 reduces the latency to avoid $(\mathrm{h})$ and freezing time during the CS (i). Bottom:

657 Normalization of the Day 1 in each group. Two-way ANOVA followed by two-stage

658 linear step-up procedure of Benjamini, Krieger and Yekutieli. Avoidance rate: $F(5$, 
$65980)=9.05$; group comparisons, Halo, Day 1 vs Day $4{ }^{* * *} P<0.001$, Day 1 vs Day $5^{* * *} P$ $660<0.001$, Day 1 vs Day $6{ }^{* \star *} P<0.001$, Day 2 vs Day $4{ }^{*} P=0.015$, Day 2 vs Day $5{ }^{* \star *} P$

$661<0.001$, Day 2 vs Day $6{ }^{* * *} P<0.001$, Day 3 vs Day $4{ }^{*} P=0.015$, Day 3 vs Day $5{ }^{* * *} P$

$662<0.001$, Day 3 vs Day $6{ }^{* * *} P<0.001$, Day 4 vs Day $6{ }^{* * *} P<0.001$, Day 5 vs Day $6{ }^{*} P$

$663=0.012$. Latency to avoid: $F(5,80)=4.52$; group comparison, Halo, Day 1 vs Day $4{ }^{*} P=$ 6640.011 , Day 1 vs Day $5^{* * *} P<0.001$, Day 1 vs Day $6{ }^{* * *} P<0.001$, Day 2 vs Day $5{ }^{*} P=$ 6650.043 , Day 2 vs Day $6{ }^{* *} P<0.001$, Day 3 vs Day $5{ }^{* *} P=0.0085$, Day 3 vs Day $6{ }^{* * *} P$ $666<0.001$, Day 4 vs Day $6{ }^{* \star} P=0.0027$. CS Freezing: $F(5,80)=1.17$; group 667 comparisons, Halo, Day 3 vs Day $6{ }^{*} P=0.012$, Day 4 vs Day $6{ }^{* *} P=0.007$, Day 5 vs 668 Day $6{ }^{*} P=0.018$. Optogenetic inhibition sessions are depicted by the yellow 669 background. Data presented mean \pm s.e.m.

671 Figure 5. pPVT ${ }^{\mathrm{D} 2 \mathrm{R}}$ projections to the NAc and the CeA flexibly bias the selection of 672 defensive behaviors.

673 a, Schematic of the viral vector strategy and optical fiber placement for optogenetic 674 silencing of pPVT ${ }^{\mathrm{D} 2}-\mathrm{NAc}$ or $\mathrm{pPVT}{ }^{\mathrm{D} 2 \mathrm{R}}-\mathrm{Ce} \mathrm{A}$ axon terminals in the same subject. b-d, 675 Avoidance rate (b), latency to avoid (c) and freezing time during the CS (d) across all 676 training sessions for both Ctl (black) and Halo (red) groups. e-g, Top: Optogenetic 677 inhibition of pPVT ${ }^{\mathrm{D} 2}-\mathrm{NAc}$ axon terminals (Day 2) decreases avoidance rate (e), 678 increases latency to avoid (f) and freezing time during the CS (g), while following 679 optogenetic inhibition of pPVT ${ }^{\mathrm{D} 2 \mathrm{R}}-\mathrm{CeA}$ axon terminals (Day 3 ) reverses these changes. 680 Bottom: Normalization of the Day 1 in each group. Two-way ANOVA followed by two681 stage linear step-up procedure of Benjamini, Krieger and Yekutieli. Avoidance rate: $F(3$, 
682 683

42) $=6.09$; group comparisons, Halo, Day 1 vs Day $2{ }^{* *} P=0.0017$, Day 2 vs Day $3{ }^{* *}$ $P=0.0072$, Day 2 vs Day $4{ }^{* *} P=0.0078$. Latency to avoid: $F(3,42)=5.56$; group comparisons, Halo, Day 1 vs Day $2{ }^{* *} P=0.0029$, Day 2 vs Day $3{ }^{*} P=0.012$, Day 2 vs Day $4{ }^{*} P=0.02$. CS Freezing: $F(3,42)=0.74$; group comparisons, Halo, Day 1 vs Day $2 P=0.086$, Day 1 vs Day $4{ }^{*} P=0.013$, Day 2 vs Day $3{ }^{*} P=0.043$. Optogenetic inhibition sessions are depicted by the yellow background. Data shown as mean \pm s.e.m.

\section{Extended Data Figure 1. pPVT ${ }^{\mathrm{D} 2 \mathrm{R}}$ projections to NAc and CeA are partially} segregated.

a, Schematic of the viral vector strategy for anterograde tracing pPVT projections. b, Representative images showing Halo-mCherry expression in neurons of the pPVT (left) and axon terminals within the NAc (middle) and the $\mathrm{CeA}$ (right). c, Schematic of the viral vector strategy for anterograde tracing $\mathrm{pPVT}{ }^{\mathrm{D} 2 \mathrm{R}}$ projections. $\mathbf{d}$, Representative images showing ChR2-YFP expression in pPVT ${ }^{\mathrm{D} 2 \mathrm{R}}$ neurons (left) and axon terminals within the NAc (middle) and the CeA (right). e, Schematic of the retrograde tracing strategy used for labeling CeA- and NAc-projecting pPVT neurons. f, Representative images showing the targets for dual-color CTB injections, green into the NAc (left) and red into the CeA (right). g, Representative images showing the retrograde labeled NAc-projecting cells (green) and CeA-projecting cells (red) are predominantly present in the D2R-labeled (grey) pPVT region. $\mathbf{h}$, Quantification of the density of CeA- and NAc-projecting neurons in the pPVT. NAc-projectors (NAc+), $1180 \pm$ 163.36; CeA-projectors (CeA+), $732.2 \pm$ 57.97; $\mathrm{n}=5$ mice; two-tailed Student's $t$-test, ${ }^{*} P=0.032$. i, Most of NAc-projecting or 
705 CeA-projecting pPVT cells express D2R protein. D2R+NAc+/NAc+, $99.21 \pm 0.22 \%$;

$706 \mathrm{D} 2 \mathrm{R}+\mathrm{CeA}+/ \mathrm{CeA}+, 99.68 \pm 0.26 \%$; two-tailed Student's $t$-test, $P=0.2 . \mathrm{j}$, Quantification

707 of the percentage of double-projecting cells in pPVT. NAc+CeA+/NAc+, $16.71 \pm 0.96 \%$;

$708 \mathrm{NAc}+\mathrm{CeA}+/ \mathrm{CeA}+, 27.18 \pm 3.39 \%$; two-tailed Student's $t$-test, ${ }^{*} P=0.018$. Data shown as 709 mean \pm s.e.m.

\section{Extended Data Figure 2. $\mathrm{PPVT}^{\mathrm{D} 2 \mathrm{R}}$ neuron activity during fear conditioning and}

712 retrieval.

a, Left: Schematic of AAV9-hSyn-Flex-GCaMP6s injection and optical fiber implantation into the pPVT of Drd2-Cre mice used for fiber photometry. Right: Optical fiber placements $(n=6$ mice). b, Experimental paradigm: Mice were conditioned with five presentations of an auditory CS (30 s) that co-terminated with a $0.5 \mathrm{~mA}$ foot shock $(2 \mathrm{~s}$; US) in a context A after experiencing four presentations of unpaired CS in habituation session. During retrieval session, eight unpaired CS presentations were delivered $24 \mathrm{~h}$ following conditioning in a context B. c-e, Summary plot of freezing behavior during the habituation (c), conditioning (d) and retrieval (e) sessions. f-h, Average calcium responses during the habituation (f), conditioning (g) and retrieval (h) sessions. CS and

722 US durations are depicted by the yellow and red backgrounds under the trace,

723 respectively. i-k, Quantification of calcium signal during pre-CS, CS, post-CS periods of

724 habituation (i), conditioning (j) and retrieval (k) sessions. AUC, One-way ANOVA

725 followed by two-stage linear step-up procedure of Benjamini, Krieger and Yekutieli.

726 Habituation: $\mathrm{n}=20$ Trials; $\mathrm{F}(2,57)=0.1$; group comparisons, pre-CS vs CS $P=0.76$,

727 pre-CS vs post-CS $P=0.89$, CS vs post-CS $P=0.66$. Conditioning: $\mathrm{n}=30$ Trials; $\mathrm{F}(2$, 
$87)=2.17$; group comparisons, pre-CS vs CS $P=0.067$, pre-CS vs post-CS $P=0.083$, CS vs post-CS $P=0.92$. Retrieval: $\mathrm{n}=48$ Trials; $\mathrm{F}(2,141)=7.7$; group comparisons, pre-CS vs CS ${ }^{* * *} P<0.001$, pre-CS vs post-CS $P=0.85$, CS vs post-CS ${ }^{* *} P=0.0012$. I, Average calcium responses during early (Trials 1-3; left) and late (Trials 4-5; right) conditioning trials. CS and US durations are depicted by the yellow and red backgrounds under the traces, respectively. $\mathbf{m}$, Quantification of calcium signal during the first $5 \mathrm{~s}$ following the onset of CS during conditioning sessions. Calcium signal significantly increased in the late trials compared with the early ones. AUC, Two-way ANOVA followed by two-stage linear step-up procedure of Benjamini, Krieger and Yekutieli, $n=12$ Trials; $F(4,112)=1.49$. Group comparisons: Early, $1 \mathrm{~s}$ vs $4 \mathrm{~s} P=$ $0.054,1 \mathrm{~s}$ vs $5 \mathrm{~s}{ }^{*} P=0.021 ;$ Late, $1 \mathrm{~s}$ vs $2 \mathrm{~s} P=0.058,1 \mathrm{~s}$ vs $3 \mathrm{~s}{ }^{* *} P=0.0043,1 \mathrm{~s}$ vs 4 $\mathrm{s}^{* * *} P<0.001,1 \mathrm{~s}$ vs $5 \mathrm{~s}{ }^{* * *} P<0.001,2 \mathrm{~s}$ vs $4 \mathrm{~s} P=0.07,2 \mathrm{~s}$ vs $5 \mathrm{~s}{ }^{* \star} P=0.0035,3 \mathrm{~s}$ vs $5 \mathrm{~s}{ }^{*} P=0.049$. $\mathbf{n}$, Calcium signal during US presentation in the late trials is higher than the early trials $(\mathrm{n}=6$ mice; two-tailed paired Student's $t$-test, $P=0.054) .0$, Top: Heatmaps showing calcium responses of Conditioning Trials 1-5 from individual subjects, respectively. CS duration is depicted by the yellow line above the heatmap. Bottom: Average calcium responses of the top panels. CS and US durations are depicted by the yellow and red backgrounds under the traces, respectively. Data shown as mean \pm s.e.m. with movement during the CS following fear memory retrieval. 
a, Left: Heatmap showing calcium responses (dF/F in Z-score) of individual trials during retrieval session after aligned by freezing percentage during the CS. CS duration is depicted by the yellow line above the heatmap. CS onset is depicted by the black dash

753 line. Right: Freezing percentage during the CS for individual trials. $\mathrm{n}=6$ mice, 8 retrieval 754 trials per mouse. b, Left: Linear regression analysis of the area under curve (AUC) of 755 calcium signal and freezing percentage during the CS for each retrieval trial $(P=0.007$, $\left.756 R^{2}=0.15\right)$. Based on the level of freezing percentage during the CS, all retrieval trials 757 are divided into three groups: Low freezing (L; Freezing < 40\%; $n=20$ trials), moderate 758 freezing $(\mathrm{M} ; 40 \% \leq$ Freezing $\leq 60 \% ; n=16$ trials $)$ and high freezing $(\mathrm{H}$; Freezing $>60 \%$; $759 \mathrm{n}=12$ trials). Right: Comparison of average calcium signal during the CS for the three 760 groups. AUC, $F(2,45)=3.3$, one-way ANOVA followed by two-stage linear step-up 761 procedure of Benjamini, Krieger and Yekutieli. Group comparisons, L vs H, * $P=0.013$.

762 c, Top: Average of calcium responses for each group. CS duration is depicted by the 763 yellow background under the trace. Middle: Average movement index for each group. 764 Bottom: Linear regression analysis of average calcium signal and movement index 765 during the CS for each group. The coefficient for high freezing trials $\left(f, R^{2}=0.44\right)$ is 766 larger than the ones for low freezing $\left(d, R^{2}=0.16\right)$ or moderate freezing $\left(e, R^{2}=0.23\right)$.

767 d, Comparison of the calcium signal (left) and movement index (right) during pre-CS, 768 CS and post-CS for the three groups. AUC, two-way ANOVA followed by two-stage 769 linear step-up procedure of Benjamini, Krieger and Yekutieli. Calcium signal: F(4, $77090)=3.37$; group comparisons, $\mathrm{M}$, pre-CS vs CS ${ }^{*} P=0.018$, CS vs post-CS ${ }^{*} P=0.01$;

$771 \mathrm{H}$, pre-CS vs CS ${ }^{* *} P<0.001$, CS vs post-CS ${ }^{* * *} P<0.001$. Movement index: $\mathrm{F}(4$, $77290)=3.6$; group comparisons, $\mathrm{L}$, pre-CS vs CS ${ }^{*} P=0.012 ; \mathrm{M}$, pre-CS vs CS ${ }^{*} P=0.023$; 
773

774

775

776

777

778

779

780

781

782

783

784

785

786

787

788

789

790

791

792

793

794

$\mathrm{H}$, pre-CS vs CS ${ }^{* *} P<0.001$, CS vs post-CS ${ }^{* *} P<0.001$. e, Average movement index in the habituation session. $\mathbf{f}$, Linear regression analysis shows poor correlation of average calcium signal and movement index during the CS for the habituation session $\left(P=0.0028, R^{2}=0.012\right) . \mathbf{g}$, Individual subjects contributing to each freezing category $(\mathrm{L}$, low freezing; $M$, moderate freezing; $H$, high freezing) in the retrieval session. Data shown as mean \pm s.e.m.

\section{Extended Data Figure 4. The individual trial activity of $\mathrm{pPVT} \mathrm{T}^{\mathrm{D} R}$ neurons during} fear retrieval session.

a, Top: Calcium responses of Retrieval Trials 1-8 from individual subjects, respectively. CS duration is depicted by the yellow line above the heatmap. Bottom: Average calcium responses of the top panels. CS duration is depicted by the yellow background under the trace. b, Quantification of calcium signal during pre-CS, CS, post-CS periods of the Retrieval Trials 1-8. AUC, One-way ANOVA followed by two-stage linear step-up procedure of Benjamini, Krieger and Yekutieli. $n=6$ subjects per trial; Trial $1, F(2,10)=$ 1.36; Trial $2, F(2,10)=4.55$, group comparisons, pre-CS vs CS ${ }^{*} P=0.018$, CS vs postCS ${ }^{*} P=0.04 ;$ Trial $3, F(2,10)=0.092 ;$ Trial $4, F(2,10)=1.55 ;$ Trial $5, F(2,10)=0.74$ Trial $6, F(2,10)=2.83 ;$ Trial $7, F(2,10)=4.94$, group comparisons, pre-CS vs CS * $P=$ 0.024 , CS vs post-CS ${ }^{*} P=0.02$; Trial $8, F(2,10)=2.71$. No significant change among other group comparison. c, Linear regression analysis of average calcium signal and movement index from the Retrieval Trials 1-8, respectively. 


\section{Extended Data Figure 5. The activity of pPVT ${ }^{\mathrm{D} 2 \mathrm{R}}$ neurons in the 2AA task, related}

to Figure 1.

a, Latency to avoid (black) and freezing time during ITI (red) across the days. Fiber photometry imaging sessions are depicted by the blue lines. b, Left: Linear regression analysis of peak calcium signal and freezing time during the CS for avoidance (blue; A; $\mathrm{R}^{2}=0.069, P=0.0043$ ) and failure trials (red; $\left.\mathrm{F} ; \mathrm{R}^{2}=0.15, P<0.001\right)$. Right: Linear regression analysis of average calcium signal and freezing time during the CS for avoidance (blue; $\mathrm{A} ; \mathrm{R}^{2}=0.17, P<0.001$ ) and failure trials (red; $\mathrm{F} ; \mathrm{R}^{2}=0.24, P<0.001$ ). c, Quantification of the latency to freezing after CS onset for avoidance and failure trials. Left: Counts of the freezing latency. Right: cumulative probability plots for the Left panel. Avoidance, $n=56$ Events; Failure, $n=109$ Events. d, Quantification of the latency to escape after CS onset for avoidance and failure trials. Left: Counts of the escape latency. Right: cumulative probability plots for the Left panel. Avoidance, $n=118$; Failure, $n=122$ Trials. Data shown as mean \pm s.e.m.

\section{Extended Data Figure 6. Optogenetic inhibition of pPVT ${ }^{\mathrm{D} 2 \mathrm{R}}$ neurons in the 2AA} task.

a, Representative image from a mouse expressing Halo-mCherry and implanted with an optical fiber in pPVT ${ }^{\mathrm{D} 2 \mathrm{R}}$ neurons. b, Optical fiber placements (Ctl, $\mathrm{n}=8$ mice; Halo, $\mathrm{n}=$ 9 mice). c, Schematic of the 2AA task. $\mathbf{d - g}$, Avoidance rate (d), latency to avoid (e) and freezing time during the CS (f) and the ITI (g) across the training days for both $\mathrm{Ctl}$ (black) and Halo (red) groups. h, Left: Quantification of the freezing time during the ITI. Right: Normalization of the Day 1 in each group. ITI Freezing in $s, F(2,30)=0.36$, two- 
way ANOVA followed by two-stage linear step-up procedure of Benjamini, Krieger and Yekutieli. No significant change among each group comparison. Optogenetic inhibition sessions are depicted by the yellow background. i, The changes in freezing behavior across test sessions are plotted as a function of changes in avoidance behavior. Day 2 vs Day 1: Halo, $P=0.23, \mathrm{R}^{2}=0.2 ; \mathrm{Ctl}, P=0.71, \mathrm{R}^{2}=0.025$. Day 3 vs Day 1 : Halo, $P=$ $0.065, R^{2}=0.41 ; \mathrm{Ctl}, P=0.27, R^{2}=0.2$. Day 3 vs Day 2: Halo, $P=0.013, R^{2}=0.61 ; C t l$, $P=0.67, \mathrm{R}^{2}=0.033$. j. Free moving distance in the open field. Ctl, $\mathrm{n}=8$ mice; Halo, $\mathrm{n}=$ 6 mice. Optogenetic inhibition duration is depicted by the yellow background. $\mathbf{k}$, Schematic of the viral vector strategy and optical fiber placement used for optogenetic silencing of pPVT ${ }^{\mathrm{D} 2 \mathrm{R}}$ neurons in the 2AA task. I, Optical fiber placements (Ctl, $n=6$ mice; Halo, $n=7)$. $\mathbf{m}-\mathbf{p}$, Avoidance rate $(m)$, latency to avoid $(n)$ and freezing time during the CS (o) and the ITI (p) across all training days in both Ctl (black) and Halo (red) groups. q-t, Left: Optogenetic inhibition of pPVT ${ }^{\mathrm{D} 2 R}$ neurons during the ITI has little effect on avoidance rate (q), the latency to avoid ( $r$ ) and freezing time during the CS (s) and the ITI (t). Right: Normalization of the Day 1 in each group. Two-way ANOVA followed by two-stage linear step-up procedure of Benjamini, Krieger and Yekutieli. Avoidance rate, $F(5,80)=9.05$; latency to avoid, $F(5,80)=4.52$; CS Freezing, $F(5$, $80)=1.17$; ITI Freezing, $F(5,80)=0.44$; no significant change among each group comparison. Optogenetic inhibition sessions are depicted by the yellow background. Data shown as mean \pm s.e.m. 
841

842

843

844

845

846

847

848

849

850

851

852

853

854

855

856

857

858

859

860

861

862

863

a, Quantification of the latency to avoid (black) and freezing time during ITI (red) across the days. Fiber photometry imaging sessions are depicted by the blue lines. $\mathbf{b}$, Left: Linear regression analysis of peak calcium signal and freezing time during the CS of avoidance (blue) and failure trials (red) (avoidance trials, $P=0.19, \mathrm{R}^{2}=0.022$; failure trials, $\left.P=0.11, R^{2}=0.02\right)$. Right: Linear regression analysis of the average calcium signal and freezing time during the CS of avoidance (blue) and failure (red) trials (avoidance trials, $P=0.012, \mathrm{R}^{2}=0.079$; failure trials, $P=0.093, \mathrm{R}^{2}=0.022$ ). Data shown as mean \pm s.e.m.

\section{Extended Data Figure 8. NAc-projecting neurons of the pPVT signal active} avoidance.

a, Schematic of the viral vector strategy and optical fiber placement used for fiber photometry imaging of NAc-projectors in pPVT. $\mathbf{b}$, Optical fiber placements $(n=5$ mice $)$.

c, Avoidance Rate (black) and freezing time during the CS (red) across all training and test sessions. Fiber photometry imaging sessions are depicted by the blue lines. d, Quantification of the latency to avoid (black) and freezing time during ITI (red) across the training and test sessions. Fiber photometry imaging sessions are depicted by the blue lines. e, Representative imaging traces from a sample subject. Individual imaging traces for avoidance (blue) and failure (red) trials. Black square pulses depict the duration of each CS presentation. Yellow and red backgrounds show the maximal duration of CS and US presentation, respectively. f, Top: Heatmaps showing individual calcium responses for all avoidance and failure trials. Bottom: Average calcium signal and CS duration of the upper panels (Avoidance trials, blue; failure trials, red; CS 
864 duration, black). g, Freezing time during the CS in avoidance trials is significantly lower 865 compared to failure trials (top), while the peak (middle) and mean (bottom) values of the calcium signal during the $\mathrm{CS}$ in avoidance trials are much higher than failure trials. Two867 tailed Student's $t$-test. Avoidance, $\mathrm{n}=85$ Trials. Failure, $\mathrm{n}=125$ Trials, ${ }^{* * *} P<0.001 . \mathbf{h}$, 868 Top: Linear regression analysis of peak calcium signal and freezing time during the CS of avoidance (blue) and failure trials (red) (avoidance trials, $P=0.67, \mathrm{R}^{2}=0.002$; failure trials, $\left.P=0.55, R^{2}=0.003\right)$. Bottom: Linear regression analysis of the average calcium

871 signal and freezing time during the CS of avoidance (blue) and failure (red) trials

872 (avoidance trials, $P=0.17, \mathrm{R}^{2}=0.02$; failure trials, $P=0.47, \mathrm{R}^{2}=0.004$ ). i,

873 Quantification of calcium signal during pre-CS, CS and post-CS periods for avoidance 874 (blue) and failure (red) trials. AUC, One-way ANOVA followed by two-stage linear step875 up procedure of Benjamini, Krieger and Yekutieli. Avoidance: $n=85$ Trials, $F(2$, $876252)=45.22$; group comparisons, pre-CS vs CS ${ }^{* *} P<0.0001$, pre-CS vs post-CS $P=$ 8770.11 , CS vs post-CS ${ }^{* * *} P<0.0001$. Failure: $\mathrm{n}=125$ Trials, $\mathrm{F}(2,372)=81.81$; group 878 879 comparisons, ${ }^{* * *} P<0.001$. j, Left: Averaged calcium responses during CS onset, CS maximal velocity, escape initiation, escape and CS freezing epochs for all avoidance 880 (blue) and failure (red) trials. Right: Quantification of calcium signal in each epoch 881 duration. AUC, two-tailed Student's $t$-test. CS: Avoidance, $\mathrm{n}=85$ Events; Failure, $\mathrm{n}=$ 882125 Events; $P=0.052$. Max. Velocity: Avoidance, $\mathrm{n}=85$ Events; Failure, $\mathrm{n}=125$

883 Events; $P=0.65$. Escape initiate: Avoidance, $\mathrm{n}=82$ Events; Failure, $\mathrm{n}=116$ Events; $884{ }^{* \star *} P<0.001$. Escape: Avoidance, $\mathrm{n}=85$ Events; Failure, $\mathrm{n}=125$ Events; ${ }^{* \star *} P<0.001$. 885 Freezing: Avoidance, $\mathrm{n}=76$ Events; Failure, $\mathrm{n}=169$ Events; $P=0.82$. Data shown as 886 mean \pm s.e.m. 
Extended Data Figure 9. Optogenetic inhibition of pPVT ${ }^{\mathrm{D} 2 \mathrm{R}}-\mathrm{NAc}$ axon terminals in the 2AA task, related to Figure 3.

a, Freezing time during the ITI across all training sessions for both Ctl (black) and Halo (red) groups. b, Left: Optogenetic inhibition of pPVT ${ }^{\mathrm{D} 2 \mathrm{R}}-\mathrm{NAc}$ axon terminals has little effect on freezing time during the the ITI. Right: Normalization of the Day 1 in each group. Two-way ANOVA followed by two-stage linear step-up procedure of Benjamini, Krieger and Yekutieli. $F(2,28)=0.149$. Optogenetic inhibition session is depicted by the yellow background. c, The changes in freezing behavior across test sessions are plotted as a function of changes in avoidance behavior. Day 2 vs Day 1: Halo, $P=$ 0.036, $\mathrm{R}^{2}=0.55 ; \mathrm{Ctl}, P=0.82, \mathrm{R}^{2}=0.0096$. Day 3 vs Day $1:$ Halo, $P=0.0022, \mathrm{R}^{2}=$ 0.81; Ctl, $P=0.036, \mathrm{R}^{2}=0.55$. Day 3 vs Day 2: Halo, $P=0.56, \mathrm{R}^{2}=0.058 ; \mathrm{Ctl}, P=$ 0.0038, $R^{2}=0.78$. Data shown as mean \pm s.e.m.

900

901

902

Extended Data Figure 10. Optogenetic inhibition of pPVT-NAc axon terminals in the 2AA task.

a, Schematic of the viral vector strategy and optical fiber placement for optogenetic silencing of pPVT-NAc axon terminals in the 2AA task. b, Optical fiber placements (Ctl, $\mathrm{n}=13$ mice; Halo, $\mathrm{n}=11$ mice). c-f, Avoidance rate (c), latency to avoid (d) and freezing time during the CS (e) and ITI (f) across all training sessions for both Ctl (black) and Halo (red) groups. g-j, Left: Optogenetic inhibition of pPVT-NAc axon terminals decreases avoidance rate (g), while increases latency to avoid (h) and freezing time during the CS (i), not the ITI (j). Right: Normalization of the Day 1 in each group. Two- 
way ANOVA followed by two-stage linear step-up procedure of Benjamini, Krieger and

911 Yekutieli. Avoidance rate: $\mathrm{F}(2,44)=4.89$; group comparisons, Halo, Day 1 vs Day $2{ }^{*} P$

$912=0.013$, Day 1 vs Day $3 P=0.062$. Latency to avoid: $F(2,44)=2.88$; group

913 comparisons, Halo, Day 1 vs Day $2{ }^{*} P<0.024$, Day 1 vs Day $3 P=0.08$. CS Freezing:

$914 F(2,44)=1.1$; group comparisons, Halo, Day 1 vs Day $2 P=0.065$. ITI Freezing: $F(2$,

$91544)=0.46$; no significant change among each group comparison. Optogenetic inhibition

916

917

918

919

920

921

922

923

924

925

926

927

928

929

930

931

932 session is depicted by the yellow background. $\mathbf{k}$, The changes in freezing behavior across test sessions are plotted as a function of changes in avoidance behavior. Day 2 vs Day 1: Halo, $P=0.0017, \mathrm{R}^{2}=0.68 ; \mathrm{Ctl}, P=0.36, \mathrm{R}^{2}=0.075$. Day 3 vs Day 1: Halo, $P=0.12, \mathrm{R}^{2}=0.25 ; \mathrm{Ctl}, P=0.36, \mathrm{R}^{2}=0.076$. Day 3 vs Day $2:$ Halo, $P=0.12, \mathrm{R}^{2}=0.25$; Ctl, $P=0.067, \mathrm{R}^{2}=0.27$. I-o, Left: Optogenetic inhibition of pPVT-NAc axon terminals during the ITI has little effect on avoidance rate $(\mathrm{I})$, the latency to avoid $(\mathrm{m})$ and freezing time during the CS (n) and the ITI (o). Right: Normalization of the Day 1 in each group. Two-way ANOVA followed by two-stage linear step-up procedure of Benjamini, Krieger and Yekutieli. Avoidance rate, $F(2,18)=0.16$; latency to avoid, $F(2,18)=0.22$; CS Freezing, $F(2,18)=0.037$; ITI Freezing, $F(2,18)=0.48$; no significant change among each group comparison. Optogenetic inhibition sessions are depicted by the yellow background. p. Free moving distance in the open field. Ctl, $n=9$ mice; Halo, $n=8$ mice. Optogenetic inhibition duration is depicted by the yellow background. Data shown as mean \pm s.e.m.

\section{Extended Data Figure 11. Fiber photometry imaging of GCaMP7s fluorescence} from the CeA terminals of pPVT ${ }^{\mathrm{D} 2 \mathrm{R}}$ neurons in the 2AA task. 
933

934

935

936

937

938

939

940

941

942

943

944

945

946

947

948

949

950

951

952

953

954

955

a, Schematic of the viral vector strategy and optical fiber placement. b, Representative images from a mouse expressing GCaMP7s in pPVT ${ }^{\mathrm{D} 2 \mathrm{R}}$ neurons (left) and implanted with an optical fiber in the $\mathrm{CeA}$ (middle). Optical fiber placements (right, $\mathrm{n}=5$ mice). c, Avoidance Rate (black) and freezing time during the CS (red) across all training and test sessions. Fiber photometry imaging sessions are depicted by the blue lines. $\mathbf{d}$, Quantification of the latency to avoid (black) and freezing time during ITI (red) across the sessions. Fiber photometry imaging sessions are depicted by the blue lines. e, Representative imaging traces from a sample subject. Individual imaging traces for avoidance (blue) and failure (red) trials. Black square pulses depict the duration of each CS presentation. Yellow and red backgrounds show the maximal duration of CS and US presentation, respectively. f, Top: Heatmaps showing individual calcium responses for all avoidance and failure trials. Bottom: Average calcium signal and CS duration of the upper panels (Avoidance trials, blue; failure trials, red; CS duration, black). g, Freezing time (top) and the peak values of the calcium signal (middle) during the CS in avoidance trials are significantly lower compared to failure trials, while the mean values of the calcium signal during the CS are similar for both groups (bottom). Two-tailed Student's $t$-test. Avoidance, $\mathrm{n}=235$ Trials. Failure, $\mathrm{n}=65$ Trials. Freezing, ${ }^{* \star *} P<0.001$. Peak Signal, ${ }^{*} P=0.026$. Mean signal, $P=0.63$. $\mathbf{h}$, Top: Linear regression analysis of peak calcium signal and freezing time during the CS of avoidance (blue) and failure trials (red) (avoidance trials, $P=0.35, \mathrm{R}^{2}=0.004$; failure trials, $P=0.12, \mathrm{R}^{2}=0.04$ ). Bottom: Linear regression analysis of the average calcium signal and freezing time during the CS of avoidance (blue) and failure (red) trials (avoidance trials, $P=0.73, \mathrm{R}^{2}=0.0005$; failure trials, $\left.P=0.082, \mathrm{R}^{2}=0.05\right)$. i, Quantification of calcium signal during pre-CS, CS 
and post-CS periods of avoidance (blue) and failure (red) trials. AUC, One-way ANOVA followed by two-stage linear step-up procedure of Benjamini, Krieger and Yekutieli. Avoidance, $n=235$ Trials, $F(2,702)=1$; Failure, $n=65$ Trials, $F(2,192)=0.025$; no significant change among each group comparison. j, Left: Averaged calcium responses during CS onset, CS maximal velocity, escape initiation, escape and CS freezing epochs in all avoidance (blue) and failure (red) trials. Right: Quantification of calcium signal in each epoch duration. AUC, two-tailed Student's $t$-test. CS: Avoidance, $n=235$ Events; Failure, $\mathrm{n}=65$ Events; $P<0.001$. Max. Velocity: Avoidance, $\mathrm{n}=234$ Events; Failure, $\mathrm{n}=62$ Events; $P=0.57$. Escape initiate: Avoidance, $\mathrm{n}=173$ Events; Failure, $\mathrm{n}$ $=101$ Events; $P=0.14$. Escape: Avoidance, $\mathrm{n}=234$ Events; Failure, $\mathrm{n}=65$ Events; $P=$ 0.42. Freezing: Avoidance, $\mathrm{n}=100$ Events; Failure, $\mathrm{n}=116$ Events; $P=0.39$. Data shown as mean \pm s.e.m.

\section{Extended Data Figure 12. Optogenetic inhibition of pPVT-CeA axon terminals in} the 2AA task.

a, Schematic of the viral vector strategy and optical fiber placement for optogenetic silencing of pPVT-CeA axon terminals in the 2AA task. b. Optical fiber placements (Ctl, $\mathrm{n}=11$ mice; Halo, $\mathrm{n}=12$ mice). $\mathbf{c}-\mathbf{f}$, Avoidance rate (c), latency to avoid (d) and freezing time during the CS (e) and ITI (f) across all training sessions for both Ctl (black) and Halo (red) groups. g-j, Left: Optogenetic inhibition of pPVT-CeA axon terminals increases avoidance rate $(\mathrm{g})$, while decreases latency to avoid (h) and freezing time during the CS (i) and the ITI (j). Right: Normalization of the Day 1 in each group. Twoway ANOVA followed by two-stage linear step-up procedure of Benjamini, Krieger and 
979 Yekutieli. Avoidance rate: $F(2,42)=3.27$; group comparisons, Ctl, Day 1 vs Day $3{ }^{*} P=$ 9800.021 , Day 2 vs Day $3{ }^{*} P=0.046$; Halo, Day 1 vs Day $2{ }^{* *} P=0.0061$, Day 1 vs Day 3 $981{ }^{*} P=0.024$. Latency to avoid: $\mathrm{F}(2,42)=5.35$; group comparisons, Ctl, Day 1 vs Day 3 $982{ }^{*} P=0.041$; Halo, Day 1 vs Day $2{ }^{* *} P=0.0049$, Day 1 vs Day $3{ }^{*} P=0.028$. CS 983 Freezing: $F(2,42)=2.77$; group comparisons, Halo, Day 1 vs Day $2{ }^{* *} P=0.0078$. ITI 984 Freezing: $F(2,42)=1.67$; group comparisons, Halo, Day 1 vs Day $2{ }^{*} P=0.038$. 985 986 987 988 989 990 991 992 Optogenetic inhibition sessions are depicted by the yellow background. $\mathbf{k}$, The changes in freezing behavior across test sessions are plotted as a function of changes in avoidance behavior. Day 2 vs Day 1: Halo, $P=0.0036, \mathrm{R}^{2}=0.59 ; \mathrm{Ctl}, P=0.82, \mathrm{R}^{2}=$ 0.0061. Day 3 vs Day 1: Halo, $P=0.28, \mathrm{R}^{2}=0.12 ; \mathrm{Ctl}, P=0.068, \mathrm{R}^{2}=0.44$. Day 3 vs Day 2: Halo, $P=0.12, \mathrm{R}^{2}=0.22 ; \mathrm{Ctl}, P=0.12, \mathrm{R}^{2}=0.24$. I-o, Left: Optogenetic inhibition of PPVT-CeA axon terminals during the ITI has little effect on avoidance rate (I), the latency to avoid (m) and freezing time during the CS (n) and the ITI (o). Right: Normalization of the Day 1 in each group. Two-way ANOVA followed by two-stage linear step-up procedure of Benjamini, Krieger and Yekutieli. Avoidance rate, F(2 ,28) $=0.0052$; latency to avoid, $F(2,28)=0.22$; CS Freezing, $F(2,28)=0.61 ; \mathrm{ITI}$ Freezing, $F(2,28)=0.086$; no significant change among each group comparison . Optogenetic inhibition sessions are depicted by the yellow background. p. Free moving distance in the open field. Ctl, $n=8$ mice; Halo, $n=9$ mice. Optogenetic inhibition 998 duration is depicted by the yellow background. 
1001

1002

1003

1004

1005

1006

1007

1008

1009

1010

1011

1012

1013

1014

1015

1016

1017

1018

1019

1020

1021

1022

1023

Extended Data Figure 13. Optogenetic inhibition of $\mathrm{pPVT}^{\mathrm{D} 2 \mathrm{R}}-\mathrm{CeA}$ axon terminals in the 2AA task, related to Figure 4.

a, Freezing time during the ITI across all training days in both Ctl (black) and Halo (red)

groups. b, Left: Optogenetic inhibition of pPVT ${ }^{\mathrm{D} 2 \mathrm{R}}-\mathrm{CeA}$ axon terminals gradually reduces freezing time during the the ITI. Right: Normalization of the Day 1 in each

group. Two-way ANOVA followed by two-stage linear step-up procedure of Benjamini, Krieger and Yekutieli. $F(5,80)=0.44$; group comparisons, Ctl, Day 1 vs Day $6{ }^{* * *} P<$ 0.001 , Day 2 vs Day $6{ }^{*} P=0.011$, Day 3 vs Day $6{ }^{* *} P=0.0074$, Day 4 vs Day $6{ }^{*} P=$ 0.048; Halo, Day 1 vs Day $6{ }^{*} P=0.017$. Optogenetic inhibition sessions are depicted by the yellow background. c, The changes in freezing behavior between Test Day 1 and other test sessions are plotted as a function of changes in avoidance behavior. Day 2 vs Day 1: Halo, $P=0.26, \mathrm{R}^{2}=0.27 ; \mathrm{Ctl}, P=0.81, \mathrm{R}^{2}=0.0092$. Day 3 vs Day $1:$ Halo, $P=$ 0.48, $\mathrm{R}^{2}=0.073 ; \mathrm{Ctl}, P=0.038, \mathrm{R}^{2}=0.48$. Day 4 vs Day $1:$ Halo, $P=0.0084, \mathrm{R}^{2}=0.65$; Ctl, $P=0.031, \mathrm{R}^{2}=0.51$. Day 5 vs Day $1:$ Halo, $P=0.031, \mathrm{R}^{2}=0.51 ; \mathrm{Ctl}, P=0.21, \mathrm{R}^{2}=$ 0.21. Day 6 vs Day 1: Halo, $P=0.032, \mathrm{R}^{2}=0.5 ; \mathrm{Ctl}, P=0.042, \mathrm{R}^{2}=0.47$. Data presented mean \pm s.e.m.

\section{Extended Data Figure 14. Optogenetic stimulation of pPVT ${ }^{\mathrm{D} 2 \mathrm{R}}-\mathrm{NAc}$ or $\mathrm{pPVT} \mathrm{T}^{\mathrm{D} 2 \mathrm{R}}$}

\section{CeA axon terminals in the 2AA task.}

a, Schematic of the viral vector strategy and optical fiber placement for optogenetic stimulating of pPVT ${ }^{\mathrm{D} 2 \mathrm{R}}-\mathrm{NAc}$ axon terminals in the 2AA task. b, Optical fiber placements (Ctl, $n=8$ mice; ChR, $n=12$ mice). c-f, Avoidance rate (c), latency to avoid (d) and freezing time during the CS (e) and ITI (f) across all training sessions for both Ctl (black) 
1024 and ChR (red) groups. g-j, Top: Optogenetic stimulation of pPVT'D2R_NAc axon

terminals increases avoidance rate $(\mathrm{g})$, while decreases latency to avoid $(\mathrm{h})$; freezing time during the CS (i) and the ITI (j) has no change. Bottom: Normalization of the Day 1 1027 in each group. Two-way ANOVA followed by two-stage linear step-up procedure of 1028 Benjamini, Krieger and Yekutieli. Avoidance rate: $F(2,36)=6.21$; group comparisons, 1029 ChR, Day 1 vs Day $2{ }^{* *} P=0.0039$, Day 1 vs Day $3{ }^{*} P=0.048$. Latency to avoid: $F(2$, $103036)=3.34$; group comparisons, ChR, Day 1 vs Day $2{ }^{*} P=0.031$, Day 1 vs Day $3 P=$ 1031 0.06. CS Freezing: $F(2,36)=0.16$; ITI Freezing: $F(2,36)=0.21$; no significant change 1032 among each group comparison. Optogenetic stimulation sessions are depicted by the 1033 yellow background. $\mathbf{k}$, The changes in freezing behavior across test sessions are 1034 plotted as a function of changes in avoidance behavior. Day 2 vs Day 1: Halo, $P=0.2$, $1035 \mathrm{R}^{2}=0.16 ; \mathrm{Ctl}, P=0.36, \mathrm{R}^{2}=0.14$. Day 3 vs Day 1 : Halo, $P=0.072, \mathrm{R}^{2}=0.28 ; \mathrm{Ctl}, P=$ $10360.22, R^{2}=0.24$. Day 3 vs Day 2: Halo, $P=0.85, R^{2}=0.0039 ; \mathrm{Ctl}, P=0.31, R^{2}=0.17 . \mathrm{I}$, 1037 Schematic of the viral vector strategy and optical fiber placement for optogenetic 1038 stimulating of pPVT ${ }^{\mathrm{D} 2 \mathrm{R}}-\mathrm{CeA}$ axon terminals in the 2AA task. $\mathbf{m}$, Optical fiber 1039 placements (Ctl, $n=7$ mice; ChR, $n=10$ mice). $\mathbf{n}-\mathbf{q}$, Avoidance rate $(n)$, latency to 1040 avoid (o) and freezing time during the CS (p) and ITI (q) across all training sessions for 1041 both Ctl (black) and ChR (red) groups. r-u, Top: Optogenetic stimulation of pPVT"2R 1042 CeA axon terminals decreases avoidance rate (r), while increases latency to avoid (s) 1043 and freezing time during the CS (t); freezing time during the ITI $(u)$ has no significant 1044 change. Bottom: Normalization of the Day 1 in each group. Two-way ANOVA followed 1045 by two-stage linear step-up procedure of Benjamini, Krieger and Yekutieli. Avoidance 1046 rate: $\mathrm{F}(2,30)=5.43$; group comparisons, Ctl, Day 1 vs Day $2{ }^{*} P=0.022$; ChR, Day 1 vs 
1047 Day $2{ }^{*} P=0.04$. Latency to avoid: $F(2,30)=1.89$; group comparisons, Ctl, Day 1 vs Day $2{ }^{*} P=0.03$; ChR, Day 1 vs Day $2{ }^{* *} P=0.0025$. CS Freezing: $F(2,30)=1.01$; $C h R$, Day 2 vs Day $3{ }^{* *} P=0.0099$. ITI Freezing: $F(2,30)=0.66$; no significant change among each group comparison. Optogenetic stimulation sessions are depicted by the yellow background. $\mathbf{v}$, The changes in freezing behavior across test sessions are plotted as a 1052 function of changes in avoidance behavior. Day 2 vs Day 1: Halo, $P=0.035, R^{2}=0.44$; Ctl, $P=0.32, \mathrm{R}^{2}=0.2$. Day 3 vs Day 1: Halo, $P=0.036, \mathrm{R}^{2}=0.44 ; \mathrm{Ctl}, P=0.079, \mathrm{R}^{2}=$ 0.49. Day 3 vs Day 2: Halo, $P=0.88, \mathrm{R}^{2}=0.003 ; \mathrm{Ctl}, P=0.19, \mathrm{R}^{2}=0.32$. Data shown as mean \pm s.e.m.

1056

Extended Data Figure 15. Optogenetic inhibition of $\mathrm{pPVT}{ }^{\mathrm{D} 2 \mathrm{R}}-\mathrm{NAc}$ or $\mathrm{pPVT}{ }^{\mathrm{D} 2 \mathrm{R}}-\mathrm{CeA}$ axon terminals in the 2AA task, related to Figure 5.

1059

a, Optical fiber placements ( $n=8$ mice per group). $b$, Freezing time during the ITI across all training sessions for both Ctl (black) and Halo (red) groups. c, Left: Optogenetic inhibition of pPVT ${ }^{\mathrm{D} 2 \mathrm{R}}-\mathrm{NAc}$ axon terminals (Day 2) decreases freezing time during the ITI, while following optogenetic inhibition of pPVT ${ }^{\mathrm{D} 2 \mathrm{R}}-\mathrm{CeA}$ axon terminals (Day 3) reverses these changes. Right: Normalization of the Day 1 in each group. Twoway ANOVA followed by two-stage linear step-up procedure of Benjamini, Krieger and Yekutieli. $F(3,42)=1.08$; Halo, Day 1 vs Day $2{ }^{*} P=0.032$. Optogenetic inhibition sessions are depicted by the yellow background. $\mathbf{d}$, The changes in freezing behavior 1067 across test sessions are plotted as a function of changes in avoidance behavior. Day 2 1068 vs Day 1: Halo, $P=0.0047, \mathrm{R}^{2}=0.76 ; \mathrm{Ctl}, P=0.11, \mathrm{R}^{2}=0.37$. Day 3 vs Day 1: Halo, $P$ $1069=0.25, \mathrm{R}^{2}=0.21 ; \mathrm{Ctl}, P=0.0044, \mathrm{R}^{2}=0.77$. Day 4 vs Day $1:$ Halo, $P=0.83, \mathrm{R}^{2}=$ $1070 \quad 0.0084 ; \mathrm{Ctl}, P=0.81, \mathrm{R}^{2}=0.01$. Day 3 vs Day 2 : Halo, $P=0.022, \mathrm{R}^{2}=0.61 ; \mathrm{Ctl}, P=$ 
$10710.064, \mathrm{R}^{2}=0.46$. Day 4 vs Day 2: Halo, $P=0.019, \mathrm{R}^{2}=0.63 ; \mathrm{Ctl}, P=0.99, \mathrm{R}^{2}<0.001$.

1072 Day 4 vs Day 3: Halo, $P=0.32, \mathrm{R}^{2}=0.16$; $\mathrm{Ctl}, P=0.27, \mathrm{R}^{2}=0.2$. Data shown as mean $1073 \pm$ s.e.m.

1074

1075 Extended Data Figure 16. Monosynaptic inputs of NAc- and CeA-projecting 1076 neurons of the pPVT.

1077 a, Schematic of the viral vector strategy to trace the inputs to NAc-projectors or CeA1078 projectors in pPVT. b, Representative images showing the rabies starter cells (Rabies1079 GFP and TVA-mCherry double-labelled cells) in pPVT neurons. c, Quantification of 1080 monosynaptic inputs to NAc-projectors or CeA-projectors in pPVT. NAc-projectors, $\mathrm{n}=$ 10813 mice; CeA-projectors, $n=2$ mice. To normalize retrogradely labeled (GFP+) cells 1082 between subjects, a connectivity index for each brain region was computed by dividing 1083 the number of retrogradely labeled cells by the number of starter cells (See Methods). 1084 The target selectivity was observed for many inputs including the medial prefrontal 1085 cortex (CeA-projectors), the agranular insular cortex (NAc-projectors), the ventromedial 1086 hypothalamus (CeA-projectors) and the substantia innominata (NAc-projectors).

\section{METHODS}

1090

\section{Mice}

1091 All procedures were performed in accordance with the Guide for the Care and Use of 1092 Laboratory Animals and were approved by the National Institute of Mental Health (NIMH) 1093 Animal Care and Use Committee. Mice used in this study were group housed under a 121094 h light-dark cycle (6 a.m. to 6 p.m. light), with food and water available ad libitum. After 
1095 surgery, mice were singly housed. Drd2-Cre mice were obtained from GENSAT (founder 1096 line ER44). In addition, we used C57BL/6NJ strain mice (The Jackson Laboratory). Both 1097 male and female mice 8-20 weeks of age were used for all experiments. Animals were 1098 randomly allocated to the different experimental conditions reported in this study.

Viral vectors

AAV9-hSyn-Flex-GCaMP6s-WPRE-SV40 was produced by the Vector Core of the 1102 University of Pennsylvania. AAV9-EF1a-DIO-eNpHR3.0-mCherry, AAV2-CaMKII2aeNpHR3.0-mCherry, AAV2-CaMKII2a-mCherry, AAV2-EF1a-DIO-hChR2(H134R)-YFP-

1104 WPRE and AAV2-EF1a-DIO-mCherry were produced by the Vector Core of the 1105 University of North Carolina. AAV5-hSyn-FLEX-ChrimsonR-tdTomato (Addgene 1106 plasmid 62723), AAV9-CAG-FLEX-tdTomato (Addgene plasmid \# 28306) and AAV91107 Syn-Flex-jGCaMP7s-WPRE (Addgene plasmid \# 104491) were purchased from Addgene. AAV2(retro)-CAG-iCre (Addgene plasmid \# 81070) was produced by Vector

1110 FLEX-RG (Addgene plasmid \# 38043) were produced by Vigene Biosciences, Inc.

1111 EnvA-SAD- $\Delta$ G-eGFP (Addgene plasmid \# 32635) was produced by the Viral Vector 1112 Core of the Salk Institute for Biological Studies. All viral vectors were stored in aliquots 1113 at $-80^{\circ} \mathrm{C}$ until use.

1116 All viral injections were performed using previously described procedures ${ }^{12}$ and an 1117 AngleTwo stereotaxic device (Leica Biosystems) at the following stereotaxic 
1118 coordinates: pPVT, $-1.60 \mathrm{~mm}$ from bregma, $0.06 \mathrm{~mm}$ lateral from midline, and $-3.30 \mathrm{~mm}$

1119 vertical from cortical surface, $6.12^{\circ}$ angle for both fiber photometry and optogenetics;

1120 NAc, $1.70 \mathrm{~mm}$ from bregma, $0.60 \mathrm{~mm}$ lateral from midline, and $-4.60 \mathrm{~mm}$ vertical from

1121 cortical surface, $9.99^{\circ}$ angle for optogenetics; CeA, $-1.42 \mathrm{~mm}$ from bregma, $2.90 \mathrm{~mm}$

1122 lateral from midline, and $-4.80 \mathrm{~mm}$ vertical from cortical surface. For fiber photometry

1123 and optogenetic experiments, an optical fiber $(400 \mu \mathrm{m}$ for photometry, Doric Lenses;

$1124200 \mu \mathrm{m}$ for optogenetics, Thorlabs) was implanted over the target immediately after viral

1125 injections (coordinates: pPVT, $-1.60 \mathrm{~mm}$ from bregma, $0.05 \mathrm{~mm}$ lateral from midline,

1126 and $-3.00 \mathrm{~mm}$ vertical from cortical surface, $6.12^{\circ}$ angle; NAc, $1.70 \mathrm{~mm}$ from bregma,

$11270.60 \mathrm{~mm}$ lateral from midline, and $-4.30 \mathrm{~mm}$ vertical from cortical surface, $9.99^{\circ}$ angle

1128 for optogenetics; CeA, $-1.42 \mathrm{~mm}$ from bregma, $2.88 \mathrm{~mm}$ lateral from midline, and -4.50

1129 mm vertical from cortical surface) and cemented using Metabond Cement System

1130 (Parkell) and Jet Brand dental acrylic (Lang Dental Manufacturing). Following all

1131 surgical procedures, animals were returned to their home cages and placed on a

1132 heating pad for $24 \mathrm{~h}$ postsurgical recovery and monitoring. Animals received

1133 subcutaneous injections with Metacam (meloxicam, 1-2 mg/kg) for analgesia and anti-

1134 inflammatory purposes. For targeting PPVT, all AAVs were injected at a total volume of

1135 approximately $1 \mu \mathrm{L}$ and were allowed 4 weeks for maximal expression. For targeting

1136 NAc, AAV2-retro-Cre virus was injected at a total volume of approximately $0.5 \mu \mathrm{L}$. For

1137 retrograde tracing of NAc-projecting and CeA-projecting pPVT cells, CTB-647 and CTB-

1138555 (1.0\% in PBS; ThermoFisher Scientific) were injected into the NAc $(0.5 \mu \mathrm{L})$ and

$1139 \mathrm{CeA}(0.3 \mu \mathrm{L})$, respectively and allowed 4 days for retrograde transport. Mice without

1140 correct targeting of optical fibers, tracers or vectors were excluded from this study. 


\section{Fiber photometry}

1143 Fiber photometry was performed as previously described ${ }^{28,29}$. Briefly, after a four-week 1144 post-surgery recovery period, mice were allowed to habituate to the fiber patch cord in 1145 their home cage for approximately $5 \mathrm{~min}$ before each behavior test. Real-time 1146 population-level GCaMP fluorescence and isosbestic autofluorescence signals were 1147 excited by the fiber photometry system (Doric Lenses) using two sinusoidally modulated $1148473 \mathrm{~nm}(211 \mathrm{~Hz})$ and $405 \mathrm{~nm}(531 \mathrm{~Hz})$ LEDs (DC4100, ThorLabs). Both LEDs were 1149 combined via a commercial Mini-cube fiber photometry apparatus (Doric Lenses) into a

1150 fiber patch-cord (400 $\mu \mathrm{m}$ core, $0.48 \mathrm{NA}$ ) connected to the brain implant in each mouse.

1151 The light intensity at the interface between the fiber tip and the animal was adjusted

1152 from 10 to $20 \mu \mathrm{W}$ (but was constant throughout each test session for each mouse).

1153 GCaMP and autofluorescence signals were collected through the same fiber and 1154 focused onto two separate photoreceivers (2151, Newport Corporation). An RZ5P fiber 1155 photometry acquisition system with Synapse software (Tucker-Davis Technologies) 1156 collected and saved real-time demodulated emission signals and behavior relevant TTL 1157 inputs of used for data analysis. For each trial, GCaMP signals $\left(F_{473} \mathrm{~nm}\right)$ were compared 1158 with autofluorescence signals $\left(F_{405 \mathrm{~nm}}\right)$ to control for movement and bleaching artefacts.

1159 Signal data was de-trended by first applying a least-squares linear fit to produce

$1160 F_{\text {fitted }} 405 \mathrm{~nm}$, and dF/F was calculated as $\left(F_{473 \mathrm{~nm}}-F_{\text {fitted } 405 \mathrm{~nm}}\right) / F_{\text {fitted }} 405 \mathrm{~nm}$. All GCaMP

1161 signal data is presented as the z-score of the dF/F from baseline (pre-CS) segments.

1162 Changes in fluorescence following behaviorally relevant events were quantified by 1163 measuring the area under the dF/F curve. 


\section{Fear conditioning}

1166 Prior to training, mice were handled and habituated to the fiber patch cord in the 1167 conditioning context. Mice were fear conditioned in a Mouse Test Cage $(18 \mathrm{~cm} \times 18 \mathrm{~cm}$ $1168 \times 30 \mathrm{~cm})$ equipped with an electrifiable floor connected to a shock generator $(\mathrm{H} 13-15$;

1169 Coulbourn instruments) and placed inside a sound attenuated cabinet (H10-24A;

1170 Coulbourn Instruments). The auditory fear conditioning procedure was carried in two

1171 separate contexts: a Context A (conditioning) and a Context B (retrieval). These

1172 contexts differed in the appearance of the walls and the texture of the floors used. In

1173 addition, for habituation and conditioning sessions, the test cage (Context A) was wiped

1174 clean with $70 \%$ ethanol in between subjects. Similarly, before each retrieval session, the

1175 test cage (Context B) was wiped clean with $0.5 \%$ acetic acid and equipped with a fruity

1176 scent to facilitate context discrimination. The cabinet was illuminated at all times and the

1177 subject's behavior was captured with a USB camera (ACT-VP-02; Coulbourn

1178 Instruments) at $3.7 \mathrm{~Hz}$ during each session. FreezeFrame software (ActiMetrics) was

1179 used to control the delivery of both 4-kHz, 75-dB tones (30 s; CS) and 0.6-mA

1180 footshocks (2 s; US). Both GCaMP signal and TTLs of CS and US presentations were

1181 monitored with the RZ5P fiber photometry acquisition system.

1182 For habituation, on day 0 a 120-s pre-stimulus interval (PSI) was followed by 4 trials of 1183 CS presentations with pseudorandom 30-60-s inter-trial interval (ITI) in Context A. On 1184 day 1 , fear conditioning was conducted in Context A. The 120 s of PSI was followed by 11855 trials of CS presentations that co-terminated with the US, with pseudorandom 30-60-S 1186 ITI. On day 2, a fear memory retrieval session was conducted in Context B. A 120 s PSI 
1187 was followed by 8 trials of unreinforced CS presentations with pseudorandom $30-120-\mathrm{s}$

1188 ITI. Freezing behavior was recorded and analyzed with FreezeFrame. For the retrieval

1189 session, the freezing data of all the trials were linearly aligned from maximus to

1190 minimum (Figure 1d, right panel). Based on the distribution, all trials were divided into

1191 three groups: Low freezing ( $\mathrm{L}$; Freezing < 40\%; $n=20$ trials), moderate freezing $(M ; 40 \%$

$1192 \leq$ Freezing $\leq 60 \% ; n=16$ trials $)$ and high freezing $(H$; Freezing $>60 \% ; n=12$ trials $)$. For

1193 all trials recorded, we calculated the area under the curve (AUC) for both calcium signal 1194 and movement.

Two-way signaled active avoidance (2AA)

1197 Mice were trained on the two-way signaled active avoidance as previously described ${ }^{16}$. 1198 Briefly, the behavioral apparatus consisted of a custom-built shuttle box $(18 \mathrm{~cm} \times 36 \mathrm{~cm}$ $1199 \times 30 \mathrm{~cm})$ that contained two identical chambers separated by a hurdle $(17.5 \mathrm{~cm} \times 6 \mathrm{~cm})$.

1200 The hurdle projected $3 \mathrm{~cm}$ above the floor and allowed mice easy access to both 1201 chambers. The floor consisted of electrifiable metal rods (H10-11M-TC-SF, Coulbourn 1202 Instruments) and was connected to a shock generator (H13-15; Coulbourn instruments).

1203 Before each subject was trained/tested, the shuttle box was wiped clean with $70 \%$ 1204 ethanol. The mouse's behavior was captured with a USB camera during each session. 1205 ANY-maze software (Stoelting) was used to control the delivery of 4-kHz, 75-dB tones 1206 (CS), 0.5-mA footshocks (US) and the TTL output for optogenetic stimulation. A speaker 1207 located on the top of the shuttle box (50 cm high) was used to deliver the CS. Subjects' 1208 movement and TTLs of CS, US and optogenetic stimulation were recorded by ANY1209 maze. 
1211 After a 5-min habituation period, mice were trained with daily sessions of $2 \mathrm{AA}$, each

1212 consisting of 30 presentations of the CS (lasting up to $15 \mathrm{~s}$ each). Trials in which

1213 subjects failed to shuttle to the adjacent chamber prior to the termination of the CS

1214 resulted in the presentation of the US (lasting up to $15 \mathrm{~s}$ each) until subjects escaped to

1215 the opposite chamber (failure trials). For trials in which subjects shuttled to the opposite 1216 chamber during the CS, the CS was abruptly terminated, and the delivery of the US

1217 prevented altogether (avoidance trials). Avoidance rate was calculated as the

1218 percentage of the number of avoidance trials over the total number of trials.

1220 For fiber photometry experiments in which the cell bodies of $\mathrm{pPVT} \mathrm{T}^{\mathrm{D} R}$ neurons were 1221 imaged (Figure 1), mice were subjected to five 2AA sessions (1 session/day) and the 1222 GCaMP signal was collected on Days 4 and 5 as described above. For optogenetic 1223 experiment of pPVT ${ }^{\mathrm{D} 2 \mathrm{R}}$ cell body (Figure 1), mice were initially trained for 5 days. If a 1224 subject did not reach $30 \%$ of avoidance rate by Day 5 , it was not used in further test 1225 sessions. Test sessions for optogenetic manipulations consisted of CS presentations 1226 (no US). In the second test session, subjects received light stimulation with a yellow 1227 light source (Ce:YAG + LED Driver; Doric Lenses) during each CS presentation (light 1228 onset was $5 \mathrm{~s}$ prior to the onset of the CS and culminated $5 \mathrm{~s}$ after the offset of the CS).

1229 The light intensity at the interface between the fiber tip and the mouse was $\sim 10 \mathrm{~mW}$. For 1230 experiments in which fiber photometry imaging of $\mathrm{pPVT} \mathrm{T}^{\mathrm{D} 2 \mathrm{R}}$ terminals and $\mathrm{pPVT} \mathrm{NAc}^{\mathrm{N}}$

1231 neurons was conducted (Figure 2, Extended Data Figure 5 and Extended Data Figure 1232 7), mice were initially trained for 3 days. GCaMP signal was collected across two 
1233 subsequent training or test sessions (referred to as Days 4-5 or test sessions) with the

1234 RZ5P fiber photometry acquisition system. For experiments involving optogenetic

1235 inhibition of pPVT ${ }^{\mathrm{D} 2}-\mathrm{NAc}$ terminals (Figure 3), mice were initially trained for 3 days. If a

1236 subject did not reach $70 \%$ of avoidance rate by Day 3 , it was not used in further testing

1237 sessions. As described above, these test sessions involved presentations of the CS

1238 alone (no US) and the subjects received light stimulation paired to CS presentations

1239 during the second test session. For experiments in which we optogenetically inhibited

1240 pPVT ${ }^{\mathrm{D} 2 \mathrm{R}}$-CeA terminals (Figure 4), we selectively screened for poor avoiders as

1241 follows: mice were initially trained for 3 days, and if a subject reached over $30 \%$ of

1242 avoidance rate by Day 3, it was not used in further test sessions. As these are

1243 essentially mice that failed to learn active avoidance, subsequent test sessions involved

1244 presentations of both the CS and the US while subjects received light stimulation paired

1245 to the CS from the second to the sixth test sessions. For experiments involving

1246 optogenetic manipulation of pPVT terminals (Extended Data Figure 6, Extended Data

1247 Figure 8 and Extended Data Figure 9), mice were initially trained for 3 days and tested

1248 with another 3 days subsequently. These test sessions involved both CS and US

1249 presentations and the subjects received light stimulation paired to CS presentations

1250 during the second test session. For experiments in which we optogenetically

1251 manipulated pPVT or its circuits during ITIs (Extended Data Figure 4, Extended Data

1252 Figure 6 and Extended Data Figure 8), mice were initially trained for 3 days and tested

1253 with another 3 days subsequently. These test sessions involved both CS and US

1254 presentations and the subjects received light stimulation paired to ITIs during the

1255 second test session. For experiments to optogenetically inhibit pPVT-NAc and pPVT- 
1256 CeA terminals within the same subjects (Figure 5), mice were initially trained for 3 days

1257 and tested with another 4 days subsequently. These test sessions involved both CS and

1258 US presentations and the subjects received light stimulation paired to CS presentations

1259 through NAc-implanted opto fibers during the second test session and CeA-implanted

1260 opto fibers during the third test session. All optogenetic experiments involved light

1261 stimulation in both mice expressing the inhibitory opsin eNpHR3.0 or excitatory opsin

1262 ChrimsonR in pPVT neurons, as well as in control mice that expressed a fluorescent

1263 reporter instead (mCherry or tdTomato).

1264

1265 Data analysis for two-way signaled active avoidance

1266 The moment-to-moment position of each mouse was determined by using an overhead

1267 camera (30 frames/s) in combination with automated tracking and trial control system

1268 (ANY-maze). We performed, post-hoc position tracking of the animal's nose and body

1269 center from video in the tracking software TopScan (CleverSys). CS and US times from

1270 ANY-maze and raw video tracking position values from TopScan were exported and

1271 analysis was performed with custom routines in the R statistical computing environment

1272 (R core Team 2019; R Foundation).

1273

1274 Missing positions up to 10 successive frames were linearly interpolated with custom

1275 routines in $\mathrm{R}$; in the very rare cases that over 10 successive frames were missing the

1276 entire video was re-tracked with optimized tracking parameters. For imaging sessions,

1277 video tracking and ANY-maze TTL pulse timestamps were zero corrected to align

1278 behavioral and calcium signal timestamps. Next, calcium signals and/or position frames 
1279 during US and CS were flagged by matching the relevant timestamps to TTL pulse 1280 times from ANY-maze, and the frame-by-frame distance traveled for the nose and body 1281 center was calculated for the tracking data. In order to minimize the effects of noise in 1282 the tracking data, we calculated the $40 \%$ quantile of the frame-by-frame distance 1283 traveled by the animal's nose and body center for each session; in all cases, this 1284 yielded a distance value of 0 or $1 \mathrm{~mm}$. This quantile value served as a movement 1285 threshold, i.e. an inter-frame distance traveled less than or equal to the quantile value 1286 was considered non-movement. We then created a binary vector and frames with 1287 coincident immobility of the nose and center body were set to 1 . Changepoint analysis ${ }^{53}$ 1288 (R package version 2.2.2, URL:https://CRAN.R-project.org/package=changepoint), with 1289 a minimum segment length of 30 video frames, was then applied to this vector. This 1290 approach allowed us to statistically determine when transitions to (and from) coincident 1291 periods of non-movement of nose and body occurred which were used as a proxy for 1292 freezing behavior. Next, each sustained bout of non-movement was isolated, and we 1293 probed whether there was any movement that lasted for $\geq 5$ consecutive video frames. 1294 If such movement did occur, we truncated the bout of immobility at the start of 1295 movement. Finally, immobility bouts with a duration $\geq 1 \mathrm{~s}$ were considered freezing. To 1296 ensure robustness of our method, we compared statistically derived event flags against 1297 manually scored videos in a subset of behavioral sessions.

1299 We isolated freezing events (see Freeze detection section above) which occurred 1300 during the CS as CS freezing and those occurred during ITI as ITI freezing. CS or ITI 1301 freezing were averaged within session and then within each group and plotted as mean 
$1302 \pm$ s.e.m. For each avoidance trial we flagged the video frame where animals first

1303 crossed the barrier and then calculated the time interval between this event and the

1304 onset of the cue, named Latency to avoid. Latency to avoid was averaged within

1305 session and then within each group and plotted as mean \pm s.e.m.

1306 As previously described (see Fiber photometry session), GCaMP data was normalized

1307 as $\mathrm{dF} / \mathrm{F}$. Next, we used the behavioral flags calculated from the video tracking to create

1308 average peri-event time histograms (PETH) time locked to the onset of the behavior

1309 events of interest, including CS onset (CS), highest movement velocity during the CS

1310 (Max. Velocity), escape or avoidance movement onset (Escape Initiate), escape or

1311 avoidance moment (Escape) and freezing onset during the CS (Freezing). Then, using

1312 the $10 \mathrm{~s}$ before each CS onset as a baseline for each trial, we calculated Z-scores from

1313 the normalized calcium signals ((Normalized signal - Mean of normalized baseline

1314 signal)/ Standard deviation of normalized baseline signal)). All trials in each session

1315 were separated into avoidance and failure trials as described above. For each trial type,

1316 the Z-score from $10 \mathrm{~s}$ before to $30 \mathrm{~s}$ after CS onset was plotted in heatmaps for all trials

1317 in all test sessions. The mean of all recorded activity for each trial type was plotted

1318 below the corresponding heatmap. We isolated the calcium signal from $2 \mathrm{~s}$ before to $2 \mathrm{~s}$

1319 after the onset of each behavior event from avoidance and failure trials separately.

1320 Using the $2 \mathrm{~s}$ before the onset as a baseline for each event, we calculated Z-scores

1321 again. Then, we calculated AUC of the Z-score from $2 \mathrm{~s}$ after the onset of each behavior

1322 event. Lastly, we plotted the mean of all signal transitions and AUC for each event type

1323 from each trial type. All 2AA photometric signals and behavioral performance were

1324 analyzed blind. 
Optogenetic inhibition of pPVT neurons and projections in an open field arena

1327

1328

1329

1330

1331

1332

1333

1334

1335

1336

1337

1338

1339

1340

1341

1342

1343

1344

1345

1346

1347

After habituating to the fiber patch cord in home cage for at least 10 min, mice were placed in a square enclosure $(50 \times 50 \mathrm{~cm})$ to roam freely for $6 \mathrm{~min}$. Mice received $2 \mathrm{~min}$ of light stimulation 2 min after the beginning of the test ( $\min 2-4)$ The light intensity at the interface between the fiber tip and the mouse was $\sim 10 \mathrm{~mW}$. The cumulative distance traveled in the open field apparatus was tracked, recorded, and quantified using ANY-maze behavioral tracking software.

\section{Monosynaptic tracing of inputs to NAc-projecting or CeA-projecting neurons of}

\section{the PVT}

To limit monosynaptic rabies tracing to NAc-projecting or CeA-projecting neurons of the PVT, AAV2(retro)-CAG-iCre was bilaterally injected into the NAc (0.5 $\mu$ l per injection) or $\mathrm{CeA}(0.3 \mu \mathrm{l}$ per injection) of C57BL/6NJ mice. Within the same surgical procedure, a virus mixture of AAV9-EF1a-FLEX-TVA-mCherry and AAV9-CAG-FLEX-RG at a 1:1 ratio was injected into the pPVT $(1.5 \mu \mathrm{l})$, followed by an injection of the pseudotyped rabies virus EnvA-SAD- $\Delta$ G-eGFP $(1.5 \mu \mathrm{l})$ in the same location of pPVT two weeks later. This viral strategy results in the ability of G-deficient rabies virus to only infect TVAreceptor expressing NAc- or CeA-projecting neurons. One week after the rabies virus injection, mouse brain tissues were collected and subjected to analysis.

Brain sections were scanned by an AxioScan (Carl Zeiss) with a $5 x$ objective. Cells were detected and quantified on Neurolnfo (MBF Bioscience). First, histological images were manually registered onto the Allen Brain Atlas (2017) on Neurolnfo. Next, linear 
1348 and non-linear transforms were applied to optimize fit. Cell coordinates were

1349 interpolated onto the registered images to generate region-specific cell counts. Starter

1350 cell count was generated by quantifying number of mCherry and GFP double positive

1351 pPVT cells. Finally, we generated a connectivity index by normalizing the fraction of

1352 retrogradely labeled $\left(\mathrm{GFP}^{+}\right)$cells for a given brain region, to the number of starter cells.

1353 Connectivity indices for each region were averaged within groups (CeA-projecting and

1354 NAc-projecting). Regions neighboring the pPVT (midline and medial thalamic nuclei)

1355 were excluded from our analyses due to the possibility of artifacts related to the injection

1356 site. All monosynaptic rabies tracing data were analyzed by a blind experimenter.

1358 Histology and immunofluorescence

1359 Animals were deeply anesthetized with euthanasia solution (Vet One) and transcardially 1360 perfused with PBS ( $\left.\mathrm{pH} 7.4,4{ }^{\circ} \mathrm{C}\right)$, followed by paraformaldehyde solution (PFA, $4 \%$ in 1361 PBS, $4{ }^{\circ} \mathrm{C}$ ). After extraction, brains were post-fixed in $4 \%$ PFA at $4{ }^{\circ} \mathrm{C}$ for a minimum of

$13622 \mathrm{~h}$, and subsequently cryoprotected by transferring to a 30\% PBS-buffered sucrose 1363 solution until brains were saturated (for over $24 \mathrm{~h}$ ). Coronal brain sections $(50 \mu \mathrm{m})$ were 1364 cut using a freezing microtome (SM 2010R, Leica). For immunofluorescence staining, 1365 Brain sections were incubated in PBS (pH 7.4) with 10\% normal goat serum (NGS) and $13660.1 \%$ Triton X-100 (Sigma Aldrich) for $1 \mathrm{~h}$, and then incubated using the following 1367 antibody (overnight, at $4{ }^{\circ} \mathrm{C}$ ): anti-D2R (1:300, mouse, Frontier Institute, D2R-Rb-Af960, 1368 validated for both species and application by producer). After four washing steps, Alexa1369 Fluor-conjugated secondary antibodies were used (Molecular Probes, 1:500, for $2 \mathrm{~h}$ at 1370 room temperature). All washes and incubations were done over a shaker at low speed. 
1371 Finally, sections were subsequently mounted onto glass slides and a coverslip was

1372 added using ProLong Diamond antifade (Thermo Fisher Scientific). Images were taken

1373 using an LSM 780 laser-scanning confocal microscope (Carl Zeiss). Image analysis and

1374 cell counting were performed using ImageJ software (Fiji, version 1.52p). Optical fiber

1375 placements for all mice included in this study are presented in Figure 1a, Figure 2c,

1376 Figure 3c, Figure 4c, Figure 5b, Extended Data Figure 4a and 4k, Extended Data Figure

1377 5b, Extended Data Figure 6b, Extended Data Figure 7b, Extended Data Figure 8b and

1378 Extended Data Figure 9b and 9m.

1379

1380

Statistical analysis

1381 All data were plotted and analyzed with OriginPro 2018 (OriginLab) and GraphPad

1382 Prism (Version 8.0.1; GraphPad Software). All data are presented as mean \pm s.e.m.

1383 There were no assumptions or corrections made before data analysis. Differences

1384 between two groups were tested with a two-tailed Student's $t$-test, where $P<0.05$ was

1385 considered significant. Differences among multiple groups were examined with analysis

1386 of variance (ANOVA, one-way and two-way repeated measures) followed by two-stage

1387 linear step-up procedure of Benjamini, Krieger and Yekutieli, where $P<0.05$ was

1388 considered significant. No statistical methods were used to predetermine sample size.

1389 For tracing experiments, the sample size is $2-5$ mice. For fiber photometry experiments,

1390 the sample size is 4-6 mice. For optogenetic experiments, the sample size is 6-13 mice.

1391 All experiments were replicated at least once. All experiments were randomized, and

1392 investigators were blinded to allocation during experiments. 


\section{Life Sciences Reporting Summary}

1395 Additional information on experimental design and materials used in our study is 1396 available in the Life Sciences Reporting Summary.

\section{Data availability}

1399

1400

1401

1402

1403

1404

1405

1406

1407

1408

1409

1410

1411

1412

1413 to Y.C.)

All the data that support the findings presented in this study are available from the corresponding author upon reasonable request.

\section{Code availability}

$\mathrm{R}$ code used to analyze active avoidance behavior and photometric signal is available from the corresponding author upon reasonable request.

\section{ACKNOWLEDGEMENTS}

We thank former NIMH postbac Nicholas Ringelberg for gathering preliminary findings that encouraged some aspects of the present study. In addition, we thank the NIMH IRP Rodent Behavioral Core for their support with the development of the custom apparatus for the 2AA task, and Drs. Mark Hoon (NICHD) and Fabricio Do Monte (UT Health) for 


\section{AUTHOR CONTRIBUTIONS}

1416 J.M. performed all experiments. M.K. assisted with histological procedures and

1417 analyzed the monosynaptic rabies tracing data. B.S.B. assisted with the monosynaptic

1418 rabies tracing experiments. J.d.H. developed custom tools for analyzing behavior and

1419 calcium signals. J.M. and J.d.H analyzed the data. Y.C. contributed to funding

1420 acquisition and writing. J.M. and M.A.P. designed the study, interpreted results, and

1421 wrote the paper. 
Figure 1

a

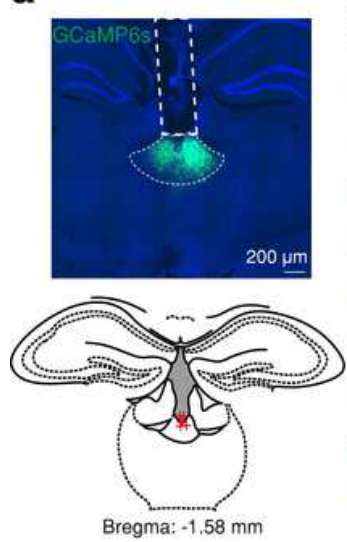

b
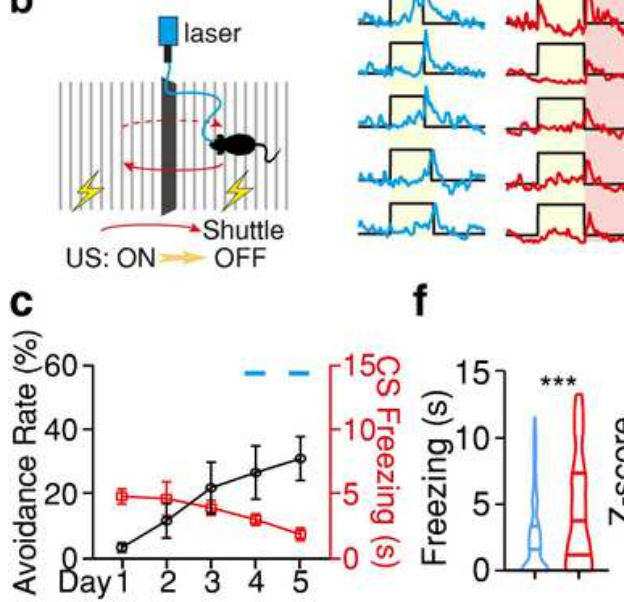

d

Avoidance Failure

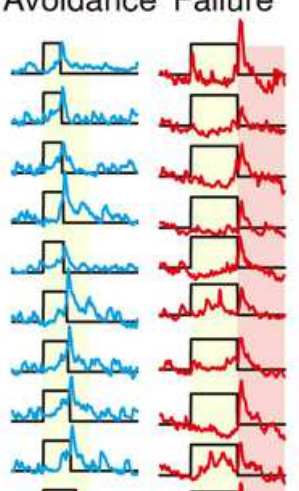

e Avoidance Trials

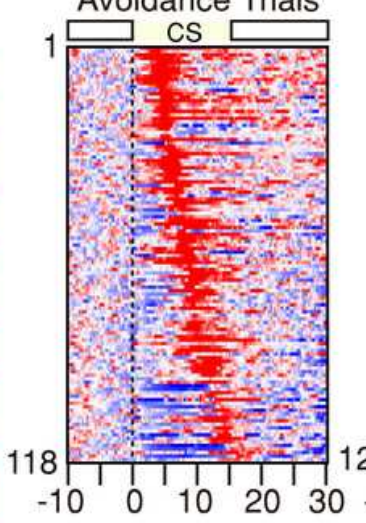

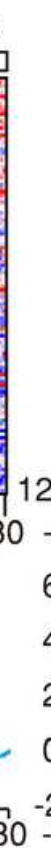
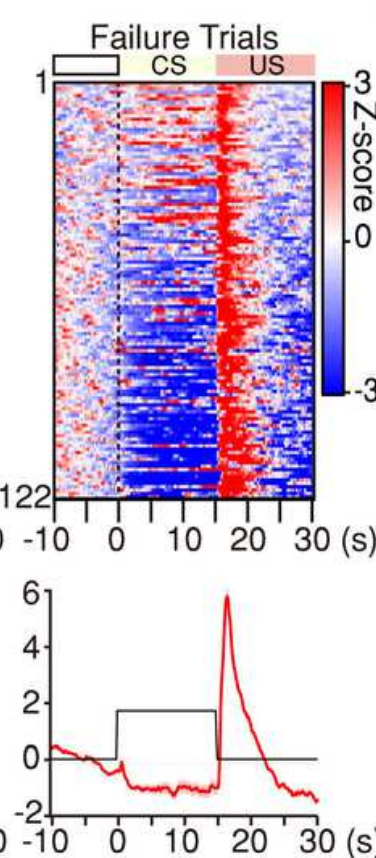

g Avoidance Failure
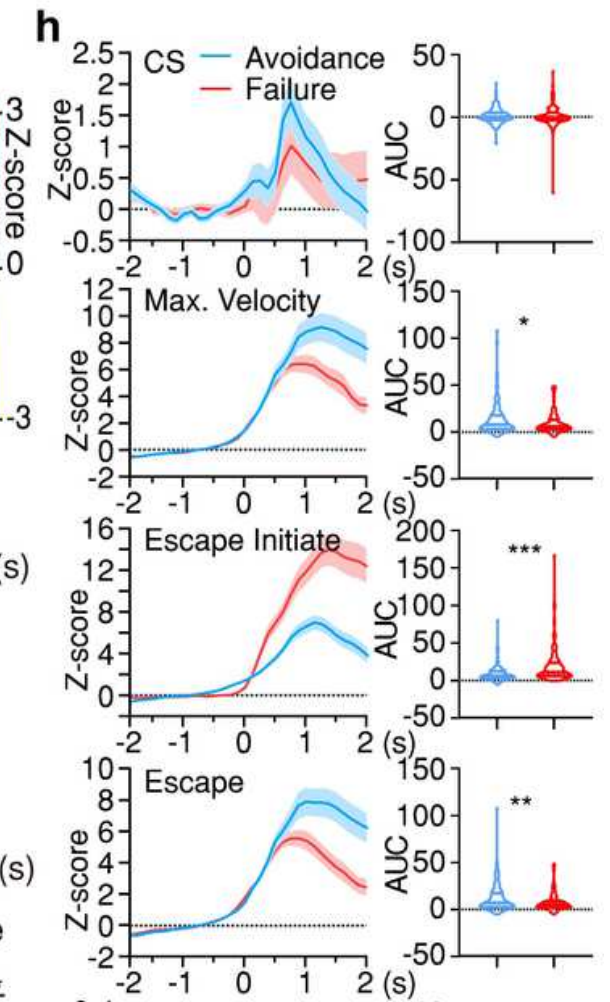

Peak Signal Mean Signal 100

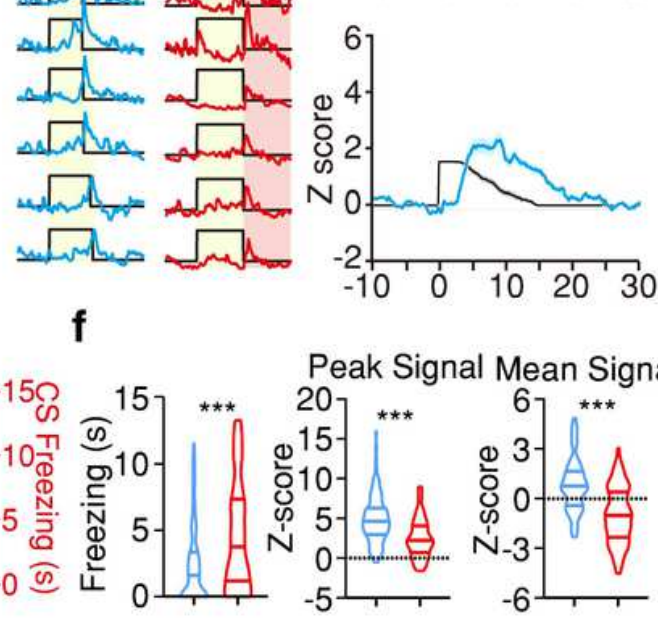

j

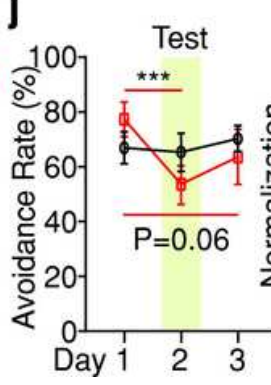

k

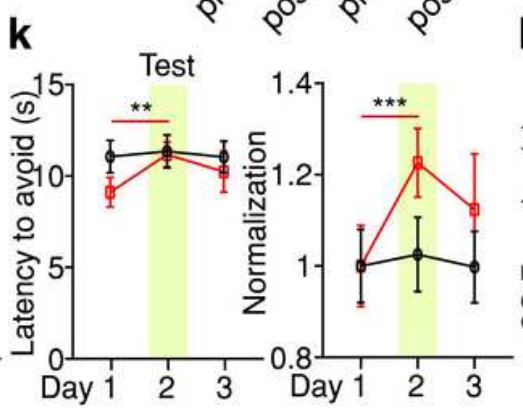

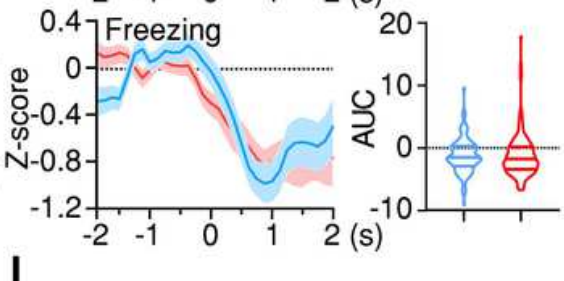

1

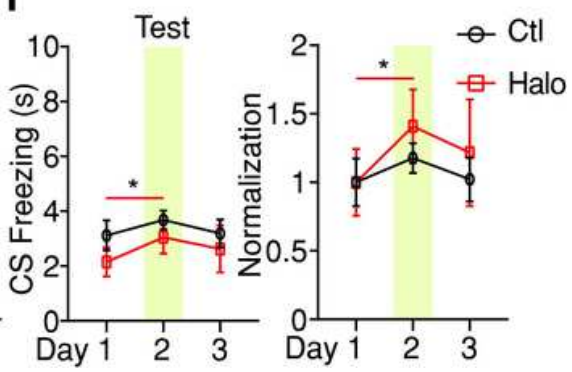

Figure 1

pPVTD2R neurons control active avoidance and are inhibited during freezing. a, Left: Representative image from a mouse expressing GCaMP6s in pPVTD2R neurons of Drd2-Cre mice and implanted with an optical fiber in the pPVT. Right: Optical fiber placements $(n=5$ mice). b, Schematic of the 2AA task. c, Avoidance Rate (black) and freezing time during the CS (red) across training (Days 1-3) and test sessions (Days 4- 5). Fiber photometry imaging sessions are depicted by the blue lines. $d$, Representative imaging traces from a sample subject. Individual imaging traces for avoidance and failure trials recorded. Bottom: Average calcium signal and CS duration of the upper panels (Avoidance trials, blue; failure trials, red; CS duration, black). $\mathrm{f}$, Freezing time during the CS (top) in avoidance trials is significantly less than failure 
trials, while the peak (middle) and mean (bottom) of calcium signals during the CS is significantly larger in avoidance trials. Avoidance, $n=118$ Trials; Failure, $n=122$ Trials; two-tailed Student's t-test. *** $P<$ 0.001. g, Quantification of calcium signal during pre-CS, CS and post-CS for avoidance (blue) and failure trials (red). AUC, One-way ANOVA followed by two-stage linear step-up procedure of Benjamini, Krieger and Yekutieli. Avoidance: $\mathrm{n}=118$ Trials; $\mathrm{F}(2,351)=75.89$; group comparisons, pre-CS vs $\mathrm{CS} * * * \mathrm{P}<0.001$, pre-CS vs post-CS $* P=0.038$, CS vs post-CS $* \star * P<0.001$. Failure: $n=122$ Trials; $F(2,363)=66.54$; group comparisons, ${ }^{\star * *} \mathrm{P}<0.001$. h, Left: Averaged calcium responses during CS onset, CS maximal velocity, escape initiation, escape and CS freezing epochs for all avoidance (blue) and failure (red) trials. Right: quantification of calcium signal in each epoch duration. AUC, two-tailed Student's t-test. CS: Avoidance, $n$ $=118$ Events; Failure, $n=122$ Events; $P=0.74$. Max. Velocity: Avoidance, $n=117$ Events; Failure, $n=122$ Events; ${ }^{*} \mathrm{P}=0.024$. Escape initiate: Avoidance, $\mathrm{n}=113$ Events; Failure, $\mathrm{n}=115$ Events; ${ }^{\star \star \star} \mathrm{P}<0.001$. Escape: Avoidance, $n=118$ Events; Failure, $n=122$ Events; $* * P=0.0064$. Freezing: Avoidance, $n=92$ Events; Failure, $n=157$ Events; $P=0.98$. i, Schematic of the viral vector strategy for optogenetic silencing of pPVTD2R neurons. j-I, Left: Optogenetic inhibition of PPVTD2R neurons significantly decreases avoidance rate $(\mathrm{j})$, while increases latency to avoid $(\mathrm{k})$ and freezing time during the CS (I). Right: Normalization of the Day 1 in each group. Ctl, $n=8$ mice; Halo, $n=9$ mice, two-way ANOVA followed by two-stage linear step-up procedure of Benjamini, Krieger and Yekutieli. Avoidance Rate: $F(2,30)=5.48$; group comparisons, Halo, Day 1 vs Day $2 * \star * P<0.001$, Day 1 vs Day $3 \mathrm{P}=0.06$. Latency to avoid: $\mathrm{F}(2$, $30)=2.48$; group comparisons, Halo, Day 1 vs Day $2 \star * P=0.0037$. CS freezing: $F(2,30)=0.19$; group comparisons, Halo, Day 1 vs Day $2 * \mathrm{P}=0.04$. Optogenetic inhibition sessions are depicted by the yellow background. Data shown as mean \pm s.e.m. 
a

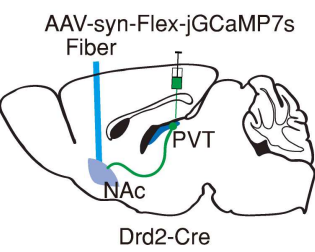

e
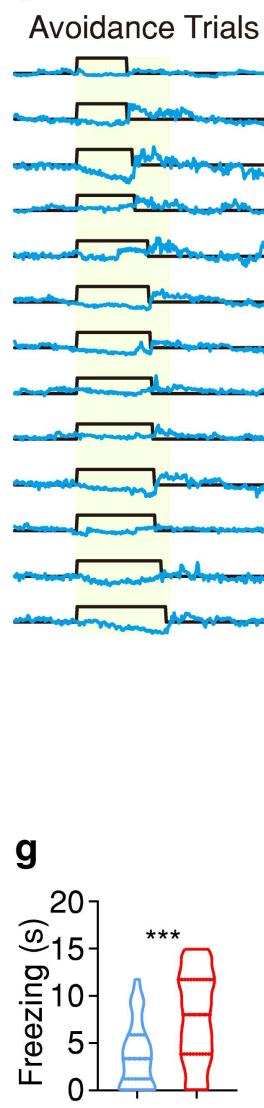

b

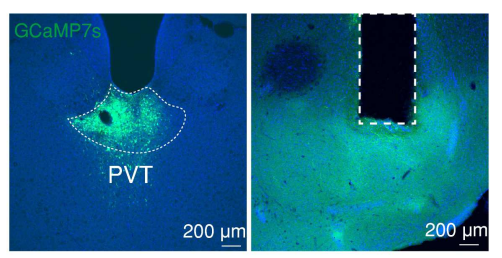

f c

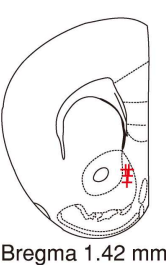

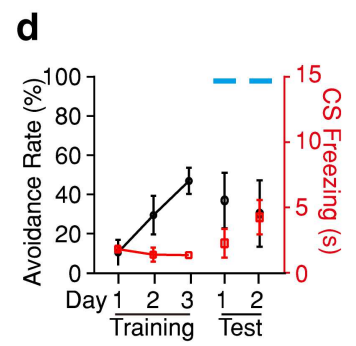

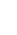
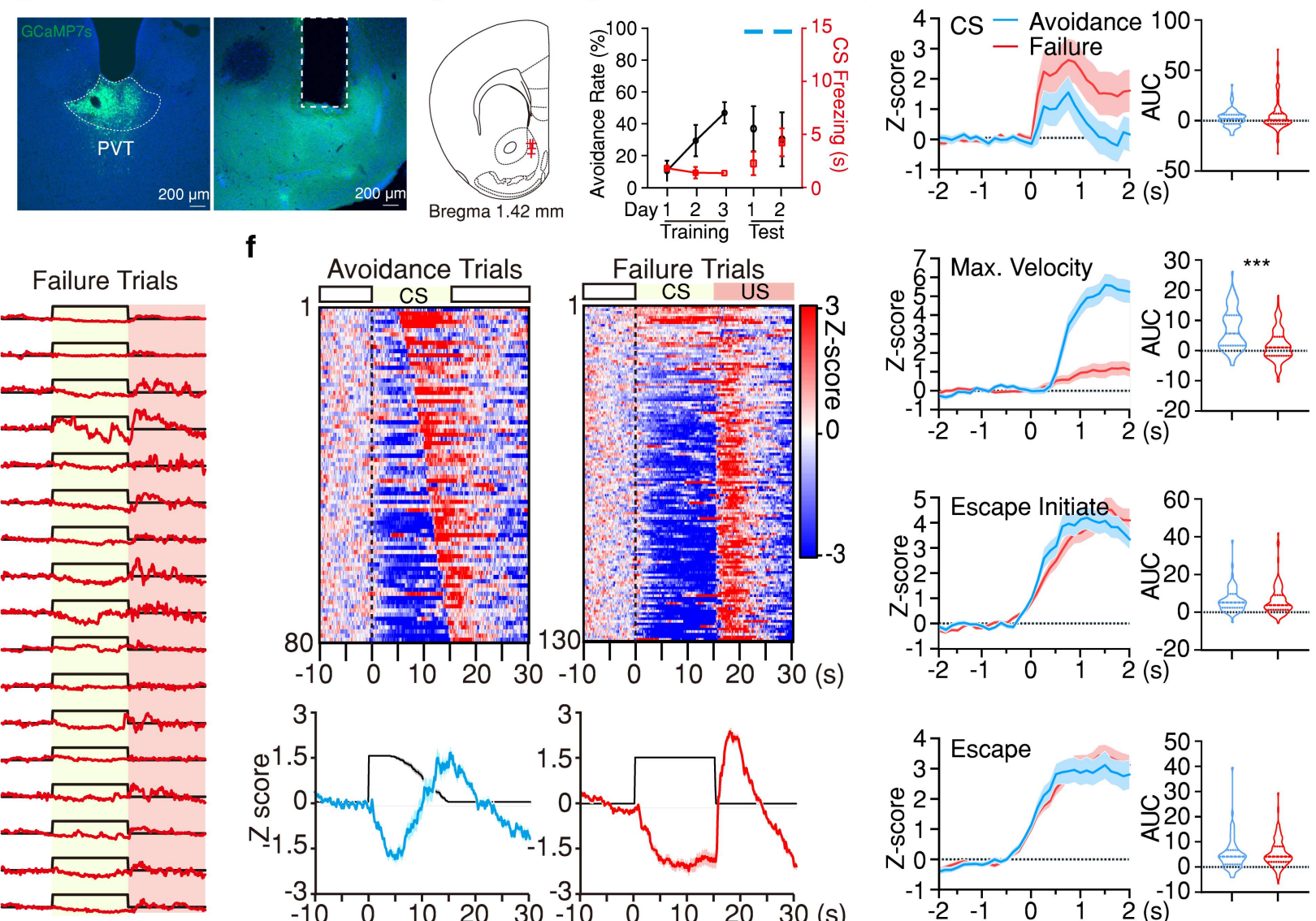

Failure Trials
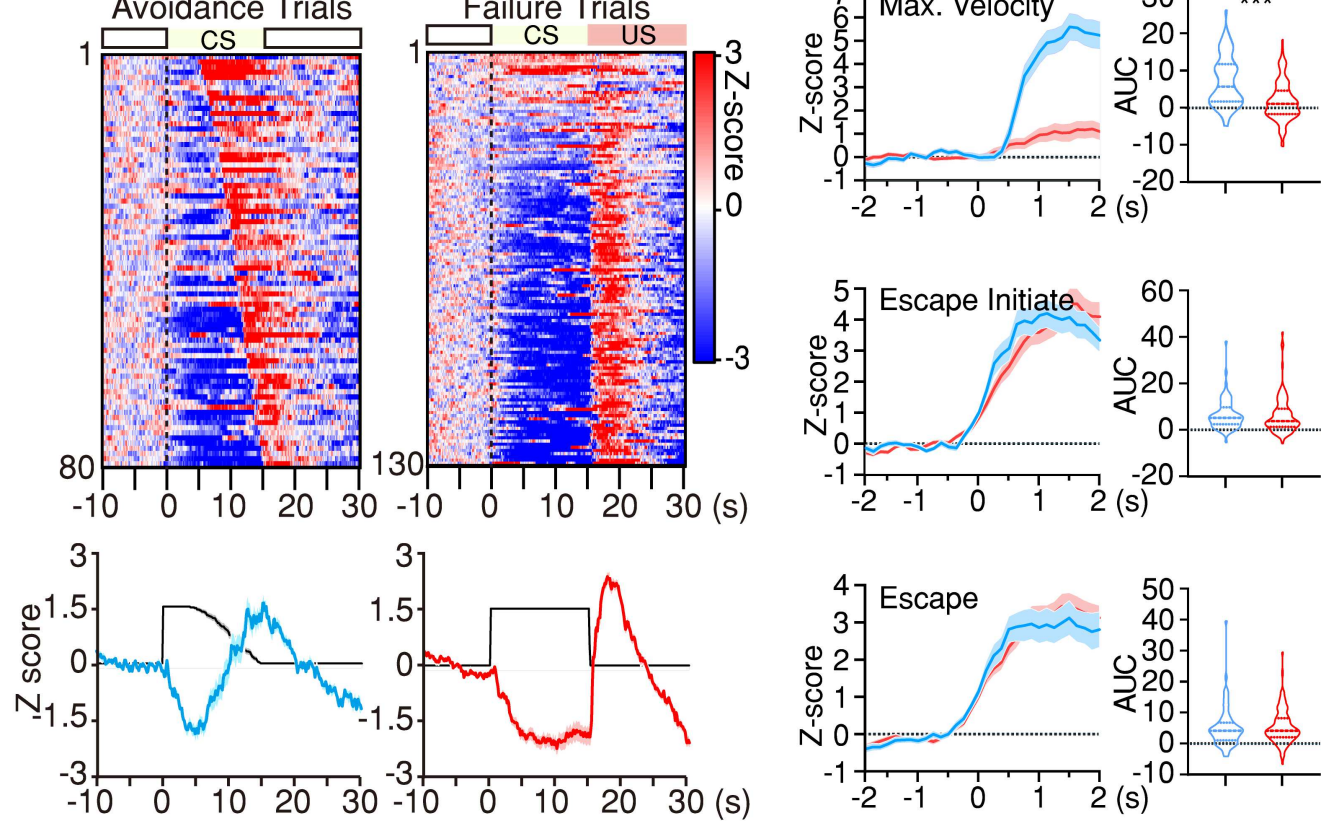

0 (s)
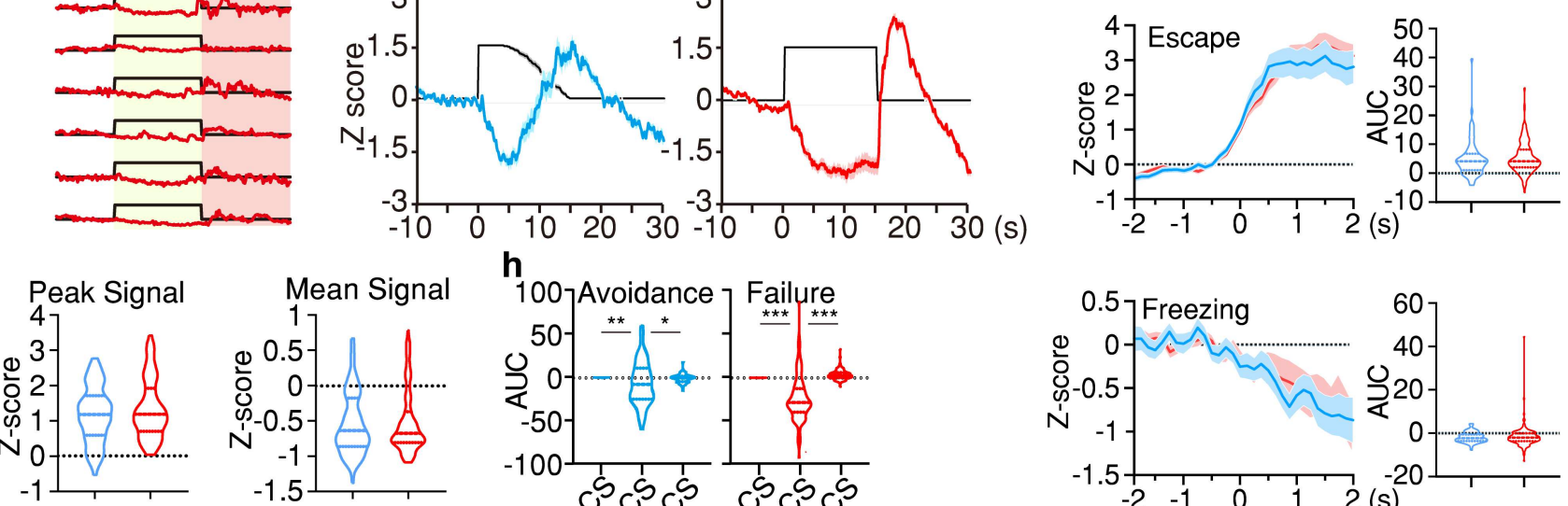

h
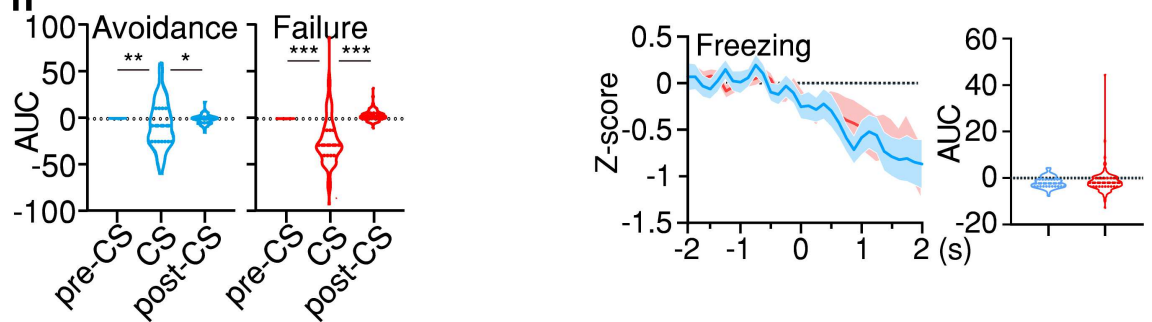

Figure 2

pPVTD2R-NAc axon terminals signal active avoidance. a, Schematic of the viral vector strategy and optical fiber placement used for fiber photometry imaging of pPVTD2R-NAc axon terminals. $b$, Representative images from a mouse expressing GCaMP7s in pPVTD2R neurons and implanted with an optical fiber in the NAc. c, Optical fiber placements $(n=4$ mice). $d$, Avoidance Rate (black) and freezing time during the CS (red) across all training and test sessions. Fiber photometry imaging sessions are depicted by the blue lines. e, Representative imaging traces from a sample subject. Individual imaging traces for avoidance (blue) and failure (red) trials. Black square pulses depict the duration of each CS presentation. Yellow and red backgrounds show the maximal duration of CS and US presentation, respectively. $f$, Top: Heatmaps showing individual calcium responses for all avoidance and failure trials. Bottom: Average calcium signal and CS duration of the upper panels (Avoidance trials, blue; failure trials, red; CS duration, black). g, Freezing time during the CS (top) in avoidance trials is significantly lower compared to failure trials, while the peak (middle) and mean (bottom) values of the calcium signal during 
the CS are similar for both groups. Two-tailed Student's t-test. Avoidance, $\mathrm{n}=80$ Trials. Failure, $\mathrm{n}=130$ Trials. Freezing, ${ }^{\star \star \star} P<0.001$. Peak Signal, $P=0.14$. Mean Signal, $P=0.75$. h, Quantification of calcium signal during pre-CS, CS and post-CS periods for avoidance (blue) and failure (red) trials. AUC, One-way ANOVA followed by two-stage linear step-up procedure of Benjamini, Krieger and Yekutieli. Avoidance: $\mathrm{n}=$ 80 Trials, $F(2,237)=4.37$; group comparisons, pre-CS vs $C S * * P=0.0065$, pre-CS vs post-CS $P=0.67$, CS vs post-CS $* \mathrm{P}=0.021$. Failure: $\mathrm{n}=130$ Trials, $\mathrm{F}(2,387)=121.2$; group comparisons, pre-CS vs $\mathrm{CS} \star \star \star P<$ 0.001 , pre-CS vs post-CS $P=0.072$, CS vs post-CS $* \star * P<0.001$. i, Left: Averaged calcium responses during CS onset, CS maximal velocity, escape initiation, escape and CS freezing epochs for all avoidance (blue) and failure (red) trials. Right: Quantification of calcium signal in each epoch duration. AUC, twotailed Student's t-test. CS: Avoidance, $n=80$ Events; Failure, $n=130$ Events; $P=0.17$. Max. Velocity: Avoidance, $n=80$ Events; Failure, $n=129$ Events; ${ }^{\star \star *} \mathrm{P}<0.001$. Escape initiate: Avoidance, $\mathrm{n}=79$ Events; Failure, $n=123$ Events; $P=0.74$. Escape: Avoidance, $n=80$ Events; Failure, $n=130$ Events; $P=0.78$. Freezing: Avoidance, $n=76$ Events; Failure, $n=175$ Events; $P=0.78$. Data shown as mean \pm s.e. $m$.

\section{Figure 3}

a

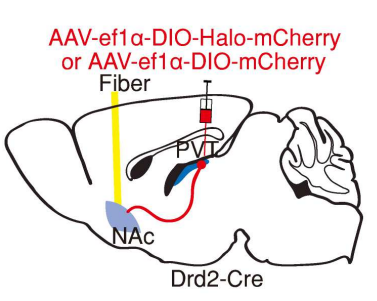

d

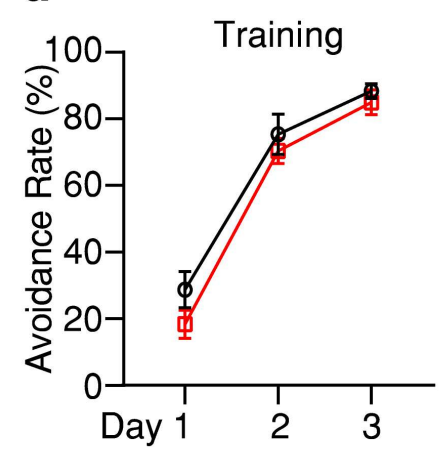

b

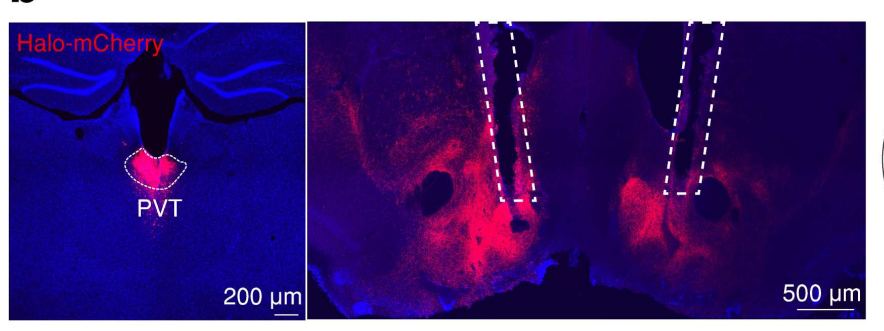

e

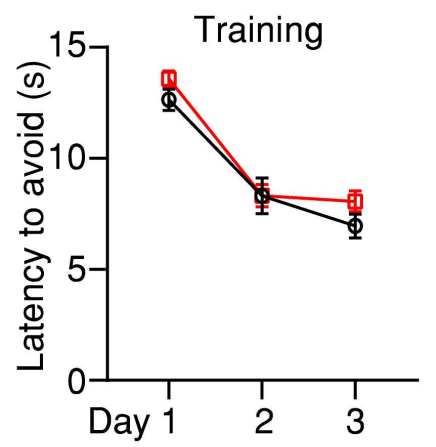

h

f g

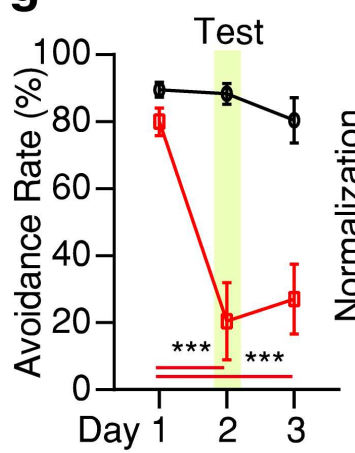

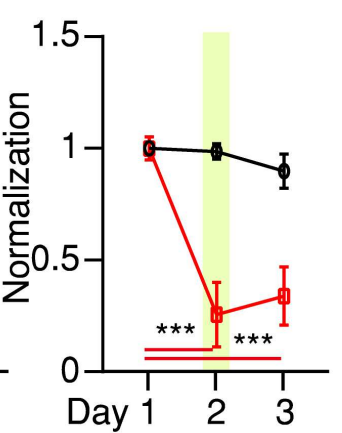

Day 123

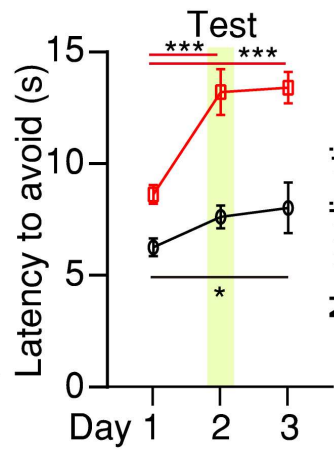

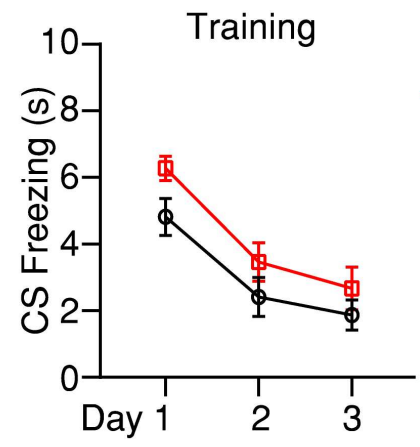

๑ CtI

๑ Halo

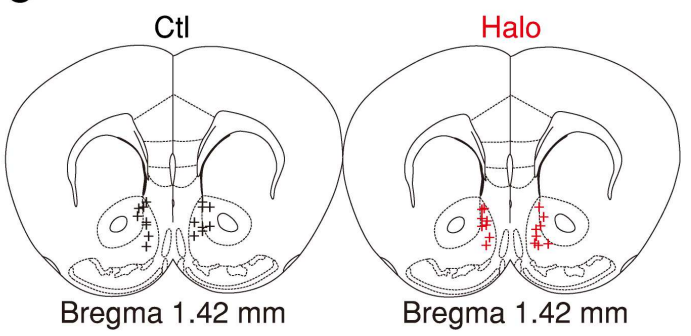

Bregma 1.42 mm

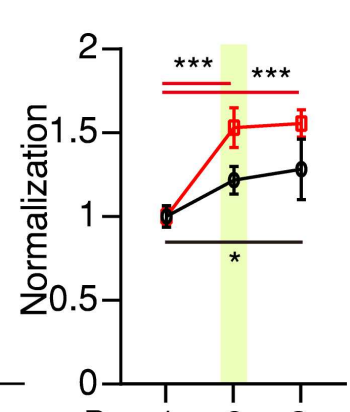

Day 123

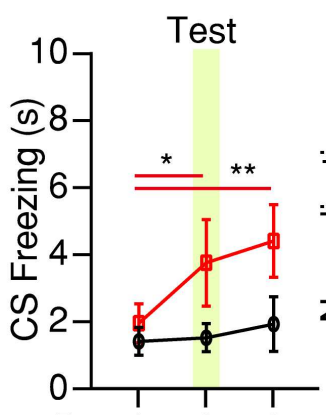

Day 123

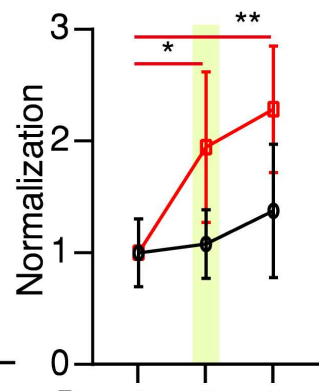

Day 123

Figure 3 
Optogenetic inhibition of pPVTD2R-NAc axon terminals decreases active avoidance and increases freezing. a, Schematic of the viral vector strategy and optical fiber placement for optogenetic silencing of pPVTD2R-NAc axon terminals in the 2AA task. b, Representative images from a mouse expressing HalomCherry in pPVTD2R neurons (left) and implanted with optical fibers in the NAc (right). c, Optical fiber placements ( $n=8$ mice per group). $d-f$, Avoidance rate (d), latency to avoid (e) and freezing time during the CS (f) across all training sessions for both Ctl (black) and Halo (red) groups. g-i, Left: Optogenetic inhibition of pPVTD2R-NAc axon terminals persistently decreases avoidance rate $(\mathrm{g})$, while increases latency to avoid (h) and freezing time during the CS (i). Right: Normalization of the Day 1 in each group. Two-way ANOVA followed by two-stage linear step-up procedure of Benjamini, Krieger and Yekutieli.

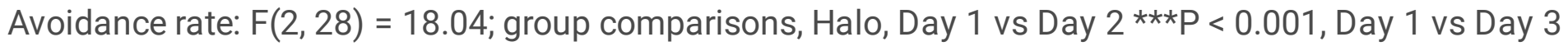
$\star \star \star P<0.001$. Latency to avoid: $F(2,28)=5.63$; group comparisons, Ctl, Day 1 vs Day $3 * P=0.028$; Halo, Day 1 vs Day $2 * \star * P<0.001$, Day 1 vs Day $3 * \star * P<0.001$. CS Freezing: $F(2,28)=1.45$; group comparisons, Halo, Day 1 vs Day $2 * P=0.049$, Day 1 vs Day $3 * * P=0.0092$. Optogenetic inhibition session is depicted by the yellow background. Data shown as mean \pm s.e.m. 

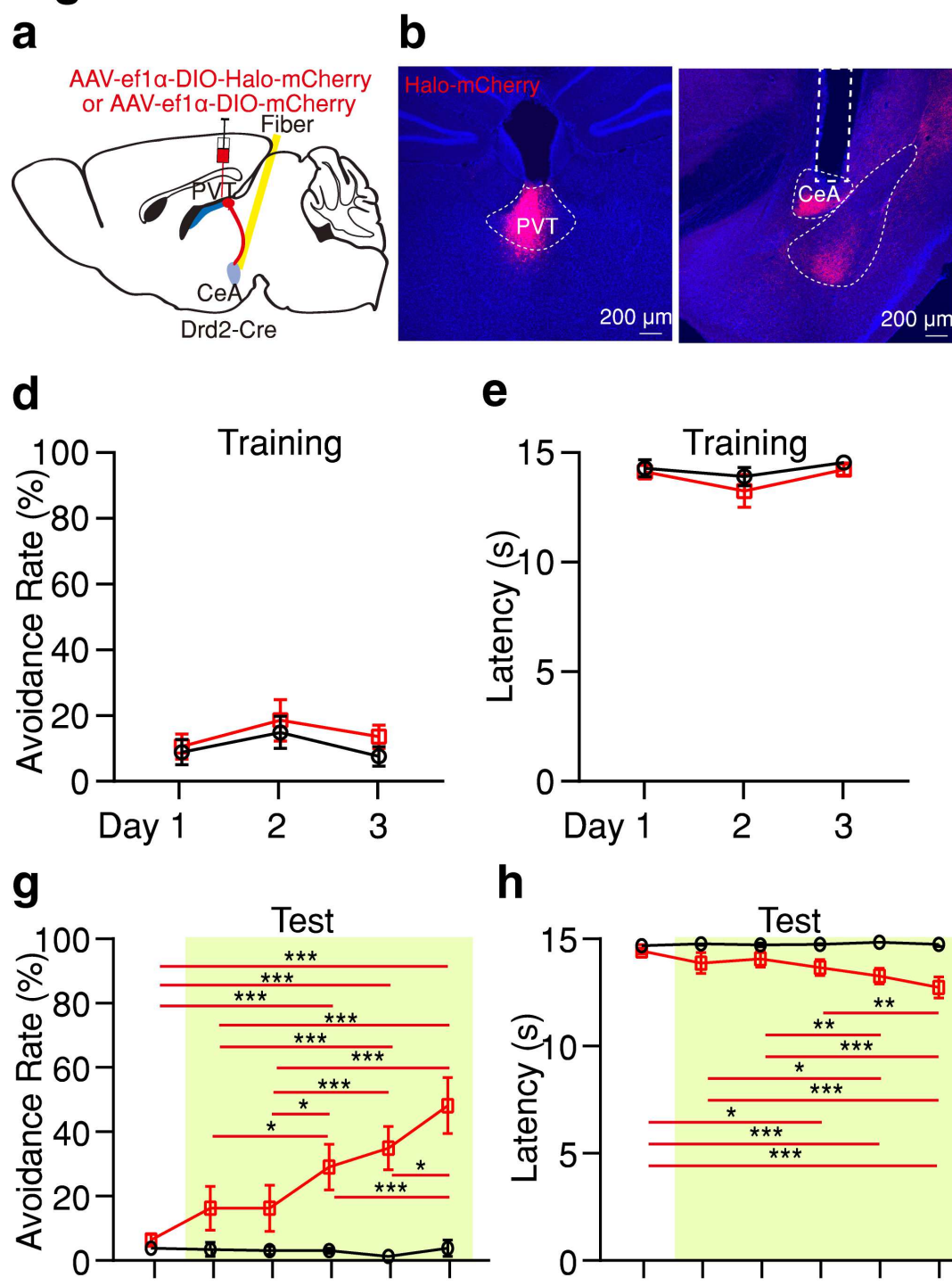

$\begin{array}{llllll}\text { Day } 1 & 2 & 3 & 4 & 5 & 6\end{array}$

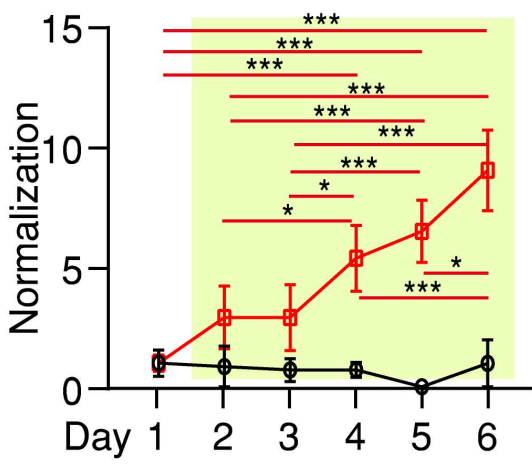

e

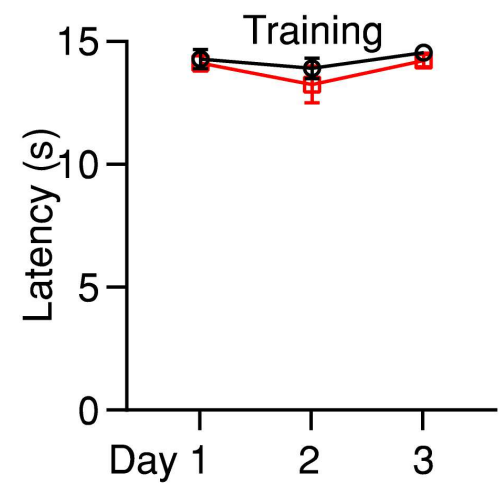

h

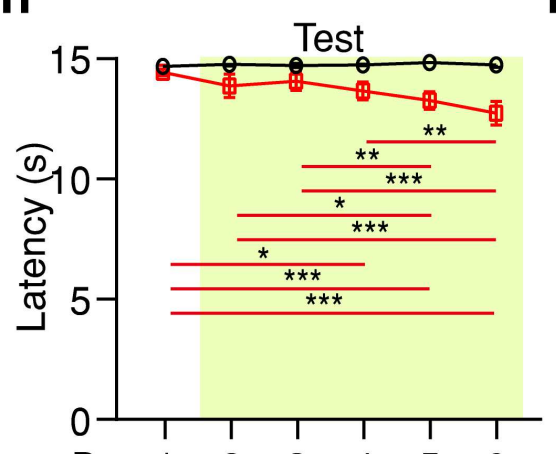

Day $1 \quad 2 \quad 3 \quad 4 \quad 5 \quad 6$

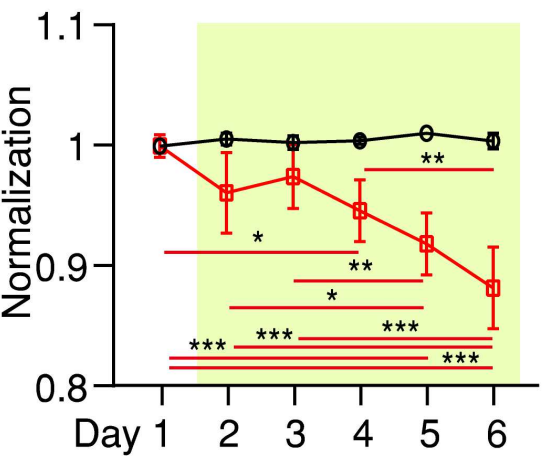

f
C
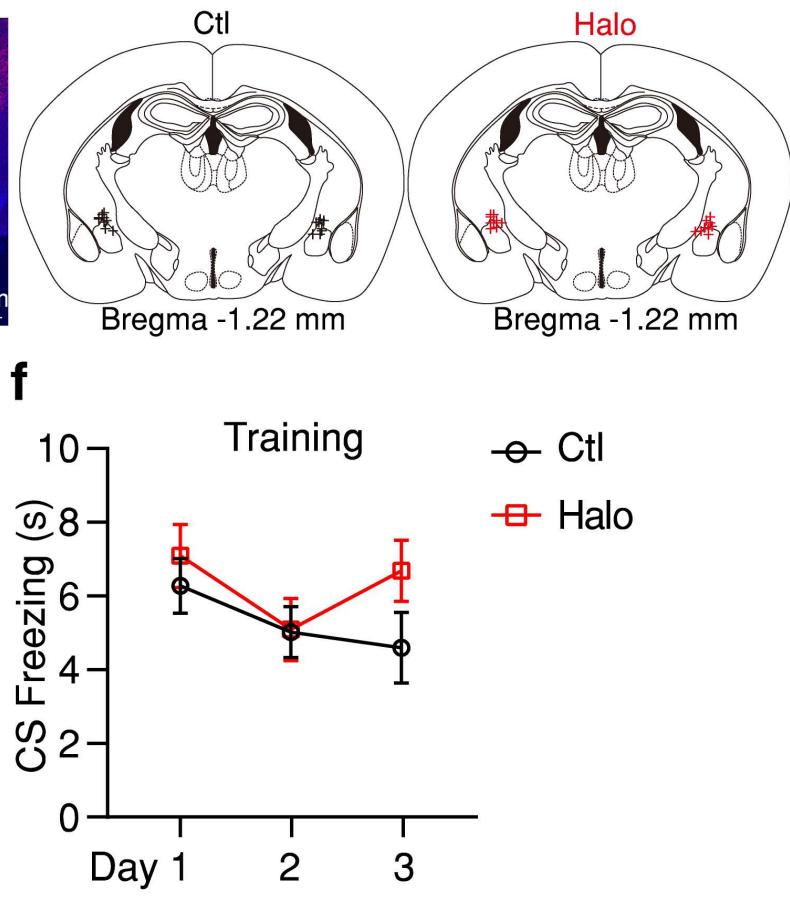

i

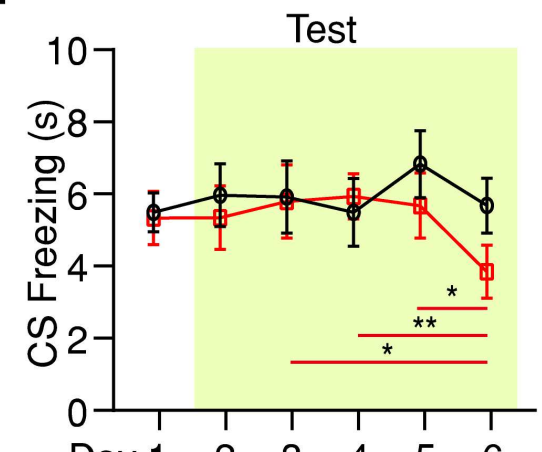

Day $1 \quad 2 \quad 3 \quad 4 \quad 5 \quad 5 \quad 6$

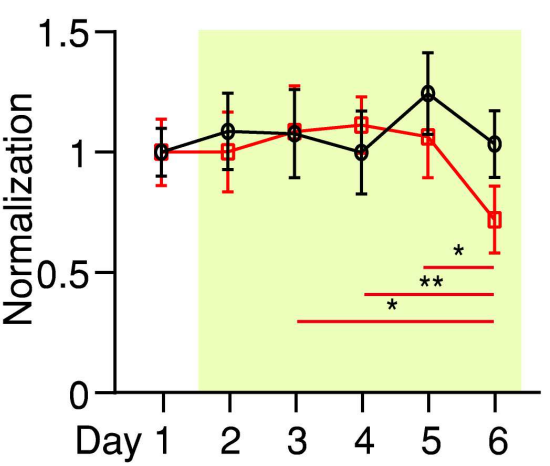

\section{Figure 4}

Optogenetic inhibition pPVTD2R-CeA axon terminals increases active avoidance and reduces freezing. a, Schematic of the viral vector strategy and optical fiber placement used for optogenetic silencing of pPVTD2R-CeA axon terminals in the 2AA task. b, Representative images from a mouse expressing HalomCherry in pPVTD2R neurons and implanted with optical fibers in the CeA. c, Optical fiber placements ( $=9$ mice per group). $d-f$, Avoidance rate (d), latency to avoid (e) and freezing time during the CS (f) across 
all training days in both Ctl (black) and Halo (red) groups. g-i, Top: Optogenetic inhibition of pPVTD2RCeA axon terminals gradually increases avoidance rate $(\mathrm{g})$ and reduces the latency to avoid $(\mathrm{h})$ and freezing time during the CS (i). Bottom: Normalization of the Day 1 in each group. Two-way ANOVA followed by two-stage linear step-up procedure of Benjamini, Krieger and Yekutieli. Avoidance rate: $F(5$, $80)=9.05$; group comparisons, Halo, Day 1 vs Day 4 *** $P<0.001$, Day 1 vs Day $5 * \star * P<0.001$, Day 1 vs Day $6{ }^{* *} P<0.001$, Day 2 vs Day $4 * P=0.015$, Day 2 vs Day $5 * \star * P<0.001$, Day 2 vs Day $6 * * *<0.001$, Day 3 vs Day $4 * P=0.015$, Day 3 vs Day $5 * \star * P<0.001$, Day 3 vs Day $6 * \star * P<0.001$, Day 4 vs Day 6 ***P $<0.001$, Day 5 vs Day $6 * P=0.012$. Latency to avoid: $F(5,80)=4.52$; group comparison, Halo, Day 1 vs Day $4 * P=0.011$, Day 1 vs Day $5 * \star * P<0.001$, Day 1 vs Day $6 * \star * P<0.001$, Day 2 vs Day $5 * P=0.043$, Day 2 vs Day $6 * \star \star P<0.001$, Day 3 vs Day $5 * * P=0.0085$, Day 3 vs Day $6 * \star * P<0.001$, Day 4 vs Day 6 $\star \star P=0.0027$. CS Freezing: $F(5,80)=1.17$; group comparisons, Halo, Day 3 vs Day $6 * P=0.012$, Day 4 vs Day $6 *{ }^{*} P=0.007$, Day 5 vs Day $6 * P=0.018$. Optogenetic inhibition sessions are depicted by the yellow background. Data presented mean \pm s.e.m.

\section{Figure 5}
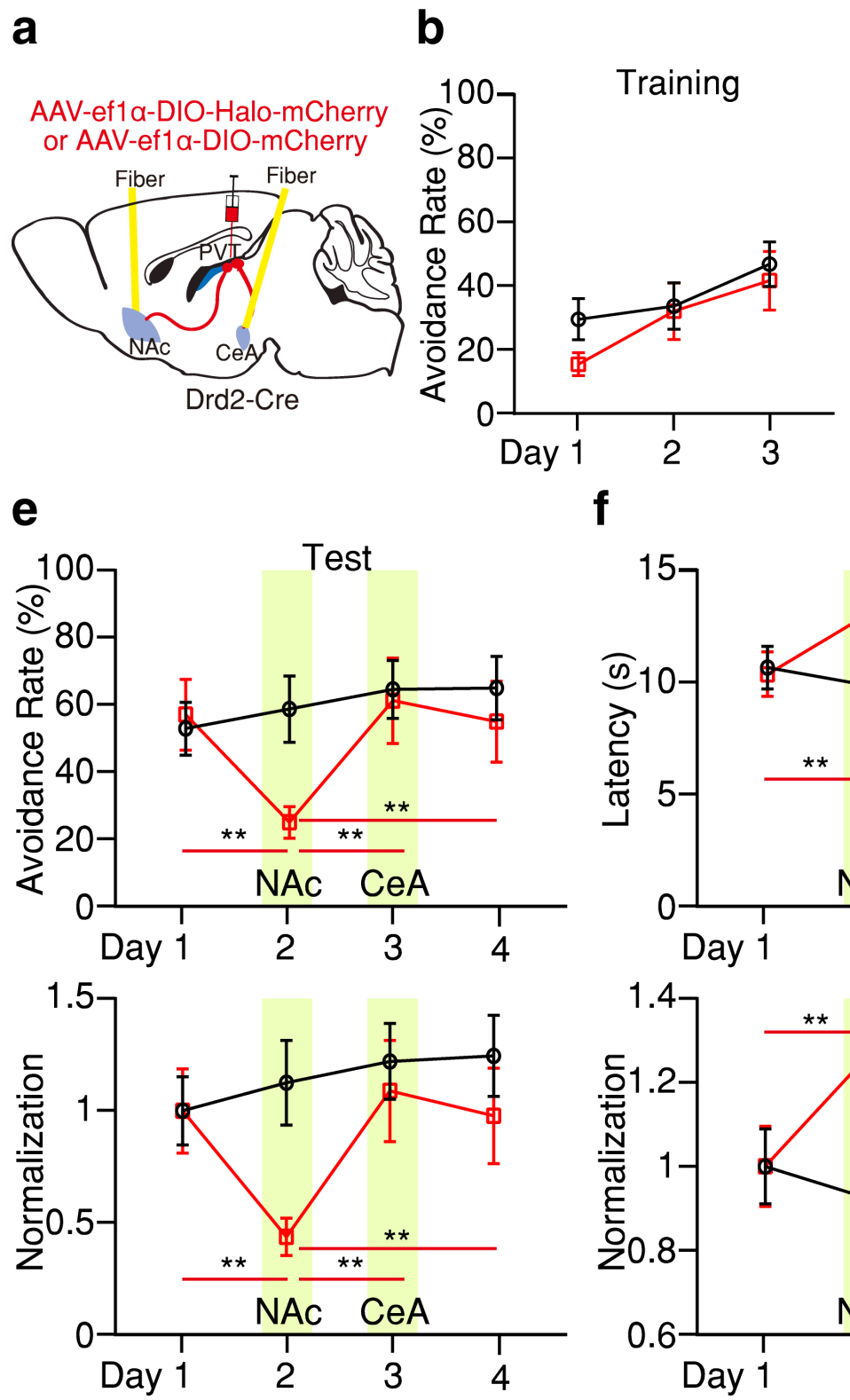

f
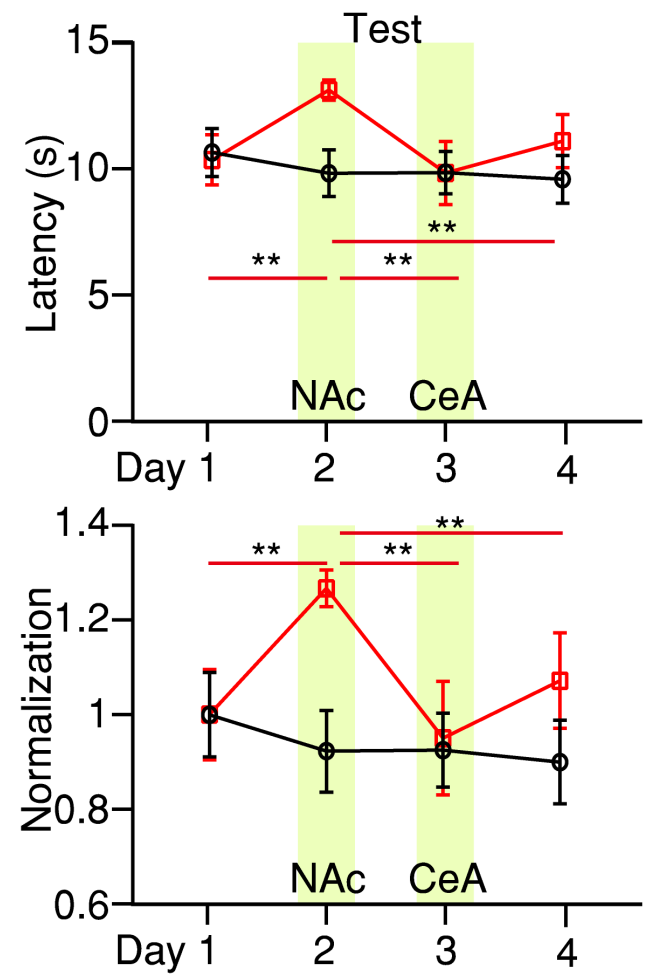

d
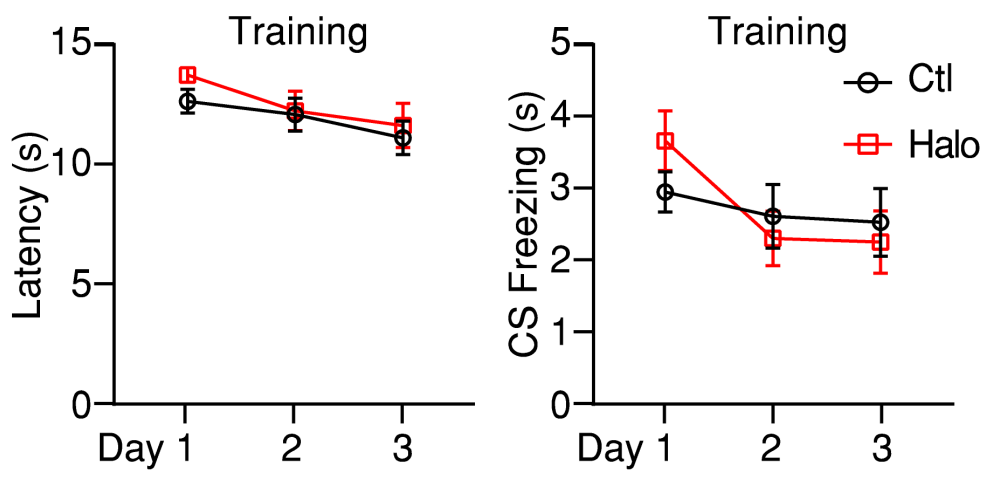

g
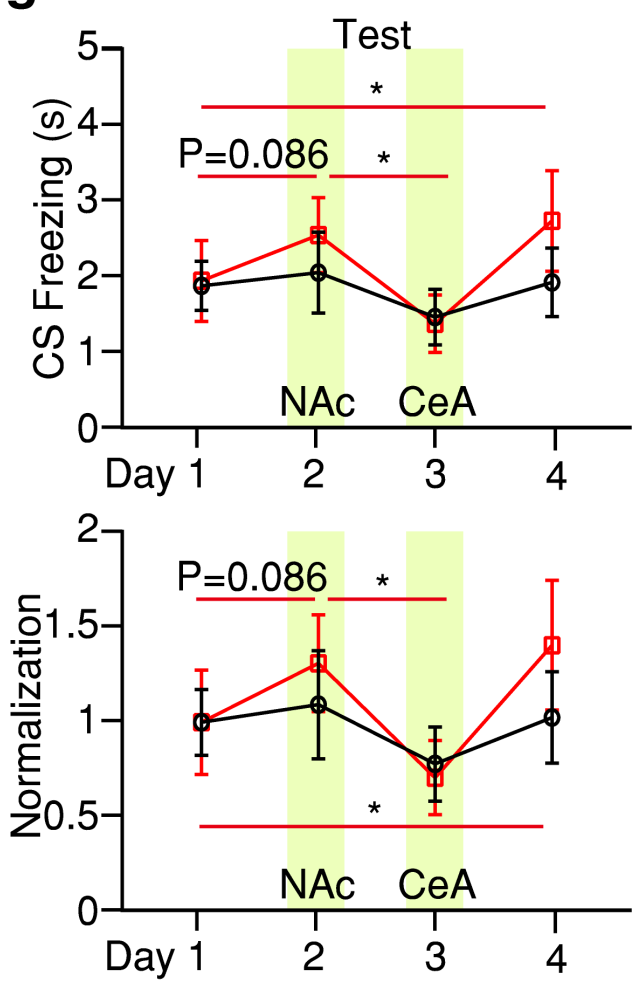


\section{Figure 5}

pPVTD2R projections to the NAc and the CeA flexibly bias the selection of defensive behaviors. a, Schematic of the viral vector strategy and optical fiber placement for optogenetic silencing of pPVTD2RNAc or pPVTD2R-CeA axon terminals in the same subject. b-d, Avoidance rate (b), latency to avoid (c) and freezing time during the CS (d) across all training sessions for both Ctl (black) and Halo (red) groups. e-g, Top: Optogenetic inhibition of pPVTD2R-NAc axon terminals (Day 2) decreases avoidance rate (e), increases latency to avoid ( $\mathrm{f}$ ) and freezing time during the CS (g), while following optogenetic inhibition of pPVTD2R-CeA axon terminals (Day 3) reverses these changes. Bottom: Normalization of the Day 1 in each group. Two-way ANOVA followed by two stage linear step-up procedure of Benjamini, Krieger and Yekutieli. Avoidance rate: $F(3,42)=6.09$; group comparisons, Halo, Day 1 vs Day $2 * \star P=0.0017$, Day 2 vs Day $3 * \star P=0.0072$, Day 2 vs Day $4 * * P=0.0078$. Latency to avoid: $F(3,42)=5.56$; group comparisons, Halo, Day 1 vs Day $2 * * P=0.0029$, Day 2 vs Day $3 * P=0.012$, Day 2 vs Day $4 * P=0.02$. CS Freezing: $F(3$, $42)=0.74$; group comparisons, Halo, Day 1 vs Day $2 \mathrm{P}=0.086$, Day 1 vs Day 4 * $P=0.013$, Day 2 vs Day

$3 * P=0.043$. Optogenetic inhibition sessions are depicted by the yellow background. Data shown as mean \pm s.e.m.

\section{Supplementary Files}

This is a list of supplementary files associated with this preprint. Click to download.

- AllSupplementaryFigures.pdf

- ExtendedDataFigure1.tif

- ExtendedDataFigure2.tif

- ExtendedDataFigure3.tif

- ExtendedDataFigure4.tif

- ExtendedDataFigure5.tif

- ExtendedDataFigure6.tif

- ExtendedDataFigure7.tif

- ExtendedDataFigure8.tif

- ExtendedDataFigure9.tif

- ExtendedDataFigure10.tif

- ExtendedDataFigure11.tif

- ExtendedDataFigure12.tif

- ExtendedDataFigure13.tif

- ExtendedDataFigure14.tif

- ExtendedDataFigure15.tif

- SupplementaryMovie1.mp4 
- ExtendedDataFigure16.tif

- SupplementaryMovie2.mp4 